\title{
Rigorous Computation of Diffusion Coefficients for Expanding Maps
}

\author{
O. Jenkinson $^{1}$ (D) M. Pollicott ${ }^{2} \cdot$ P. Vytnova ${ }^{2}$
}

Received: 31 August 2017 / Accepted: 21 November 2017 / Published online: 30 November 2017

(C) The Author(s) 2017. This article is an open access publication

\begin{abstract}
For real analytic expanding interval maps, a novel method is given for rigorously approximating the diffusion coefficient of real analytic observables. As a theoretical algorithm, our approximation scheme is shown to give quadratic exponential convergence to the diffusion coefficient. The method for converting this rapid convergence into explicit high precision rigorous bounds is illustrated in the setting of Lanford's map $x \mapsto 2 x+\frac{1}{2} x(1-x)$ $(\bmod 1)$.
\end{abstract}

Keywords Diffusion coefficient · Expanding map · Ergodic theory · Dynamical Systems

\section{Introduction}

For a real analytic ${ }^{1}$ expanding interval map $T: X \rightarrow X$ with absolutely continuous invariant probability measure $\mu$, and a real analytic function $g: X \rightarrow \mathbb{R}$, the corresponding diffusion coefficient (or variance) is the quantity $\sigma_{\mu}^{2}(g)$ defined by

$$
\sigma_{\mu}^{2}(g)=\lim _{n \rightarrow \infty} \frac{1}{n} \int\left(\sum_{i=0}^{n-1} g \circ T^{i}-n \int g d \mu\right)^{2} d \mu .
$$

\footnotetext{
${ }^{1}$ By convention we say that $T$ is real analytic whenever it is piecewise real analytic, i.e. the interval $X$ admits a partition into intervals, with $T$ real analytic on each partition piece.

O. Jenkinson

o.jenkinson@qmul.ac.uk

M. Pollicott

M.Pollicott@warwick.ac.uk

P. Vytnova

P.Vytnova@warwick.ac.uk

1 Queen Mary University of London, London, UK

2 University of Warwick, Coventry, UK
} 
The quantity $\sigma_{\mu}^{2}(g)$ plays a role in the central limit theorem: it is well known (see e.g. [11]) that provided $g$ is not equal to a coboundary plus a constant then

$$
\frac{1}{\sqrt{n}}\left(\sum_{i=0}^{n-1} g \circ T^{i}-n \int g d \mu\right)
$$

converges in law to a normal distribution with mean zero and variance $\sigma_{\mu}^{2}(g)>0$. A difficult problem of practical interest is to calculate, or to approximate, the diffusion coefficient $\sigma_{\mu}^{2}(g)$, noting that (1) is only rarely amenable to direct evaluation. Bahsoun et al. [2] recently gave a method for the rigorous approximation of diffusion coefficients, including error bounds, based on Ulam's method. They illustrated this approach with the particular map

$$
T(x)=2 x+\frac{1}{2} x(1-x) \quad(\bmod 1)
$$

introduced by Lanford [8], and the function $g(x)=x^{2}$, showing that $0.3458 \leq \sigma_{\mu}^{2}(g) \leq$ 0.4152 .

In this paper we develop an alternative algorithm for approximating diffusion coefficients of expanding interval maps. In general the method uses the periodic points of $T$, and exploits the real analyticity of the map $T$ and the function $g$. The method gives highly accurate approximations to the diffusion coefficient, both at the level of a theoretical algorithm converging with a given asymptotic speed (namely quadratic exponential convergence, as described in Theorem 1 below), and, most importantly, at the level of completely rigorous certified error bounds (see Theorems 2 and 3). The real analyticity assumptions will be crucial in establishing both the theoretical asymptotics and the concrete error bounds, since explicit use is made of the holomorphic extensions of the function $g$ and (the inverse branches of) the map $T$ to certain regions of the complex plane. The general asymptotic speed of our algorithm is as follows:

Theorem 1 Let $T: X \rightarrow X$ be a real analytic expanding interval map with absolutely continuous invariant probability measure $\mu$, and suppose $g: X \rightarrow \mathbb{R}$ is real analytic. There exists a sequence $\sigma_{n}^{2} \rightarrow \sigma_{\mu}^{2}(g)$, where each $\sigma_{n}^{2}$ can be explicitly computed in terms of periodic points of period up to $n$. The rate of convergence is quadratic exponential, in the sense that there exist constants $C>0$ and $\kappa \in(0,1)$ such that

$$
\left|\sigma_{\mu}^{2}(g)-\sigma_{n}^{2}\right| \leq C \kappa^{n^{2}} \text { for all } n \in \mathbb{N} .
$$

The constants $C$ and $\kappa$ of Theorem 1 can be rendered explicit, a procedure which involves consideration of holomorphic extensions to regions in the complex plane. A more challenging problem, in the context of a specific map $T$ and function $g$, is to establish effective error bounds on $\left|\sigma_{\mu}^{2}(g)-\sigma_{n}^{2}\right|$, preferably of very high accuracy; a key purpose of this article is to show that in such practical settings there is considerable scope for sharpening our optimal version of the simple asymptotic form (3) so as to obtain effective high quality bounds on the diffusion coefficient. As a model case we shall orient our discussion of this problem around the specific example considered in [2], namely Lanford's map $T$, and the function $g(x)=x^{2}$, both of which are real analytic; henceforth we refer to this as the model problem. The problem of obtaining high accuracy rigorous estimates on $\sigma_{\mu}^{2}(g)$ involves both theoretical and computer programming elements, and any proof of such bounds will invariably be computer-assisted. As a starting point we note that, using only a modern desktop computer, it is possible to 
locate all the periodic points of the Lanford map $T$ up to period $P$, for some ${ }^{2} 20 \leq P \leq 30$. Choosing maximum period $P=25$ yields the sequence of approximations to $\sigma_{\mu}^{2}(g)$ given in Table 2, which at the level of non-rigorous empirical observation suggests that

$$
\sigma_{\mu}^{2}(g)=0.36010948619916067289882418682857674924166999779722 \pm 10^{-50},
$$

and indeed a more optimistic interpretation of Table 2 suggests the slightly more accurate $\sigma_{\mu}^{2}(g)=0.3601094861991606728988241868285767492416699977972288644 \pm 10^{-55}$.

The task is to now harness these computed approximate values $\sigma_{n}^{2}$ (and in particular the last of these computed approximations, $\sigma_{P}^{2}$ ) so as to produce a fully rigorous approximation to $\sigma_{\mu}^{2}(g)$, together with an error bound. Any naive expectation that the theoretical asymptotic (3), together with specific values for $\kappa$ and $C$, would automatically yield an effective error bound on $\left|\sigma_{\mu}^{2}(g)-\sigma_{n}^{2}\right|$ is tempered by the realisation that, for the model problem, $\kappa$ is reasonably close to the value ${ }^{3} 1$, and $C$ is extremely large. ${ }^{4}$ Although, as noted above, the value $P=25$ is deemed to be the maximum such that all $\left(\sigma_{n}^{2}\right)_{n=1}^{P}$ can be explicitly evaluated, a finer analysis ${ }^{5}$ of the estimates yielding the asymptotic (3) suggests that a good quality rigorous effective estimate on $\sigma_{\mu}^{2}(g)$ remains out of reach for $P \leq 30$.

In order to obtain high quality effective estimates on $\left|\sigma_{\mu}^{2}(g)-\sigma_{n}^{2}\right|$ we therefore develop a hybrid approach, consisting of three distinct types of computation, the first type being the exact evaluation of $\sigma_{n}^{2}$ (see Sect. 3 for the formulae defining $\sigma_{n}^{2}$ ) for all sufficiently

${ }^{2}$ In general the specific value of $P$ will depend on available hardware, on the computer programming implementation of our algorithm, and on the time available to make the computation. For the Lanford map $T$ we found it possible to locate points up to period 20 in less than an hour, while locating points of period up to 25 took around a day (computations were performed in an arbitrary precision environment, giving several hundred correct decimal digits); note that since $T$ is a 2-branch map, incrementing the maximum period by one entails an approximate doubling of the computer run time.

3 For any two branch expanding map, our techniques yield a value of $\kappa$ lying in the range $\left[2^{-1 / 2}, 1\right)$, while for the Lanford map itself our optimal value is $\kappa \approx 0.927734$ (this is the square root of the quantity $\theta$ defined in (57)). Note that although the term $\kappa^{n^{2}}$ is approximately $4.3 \times 10^{-21}$ when $n=25$, the value of $C$ in (3) is too large for the asympotic estimate $\left|\sigma_{\mu}^{2}(g)-\sigma_{n}^{2}\right| \leq C \kappa^{n^{2}}$ to be effectively used until $n$ is significantly larger (and, crucially, above the maximum value of $n$ for which all $2^{n}$ period- $n$ points can be located using the computational resources at our disposal).

4 The size of $C$ will depend on $\kappa$, and $C$ becomes larger the closer $\kappa$ is chosen to the optimal value of approximately 0.927734 (see Footnote 3 ). As an indication of its order of magnitude, we use the fact (see Footnote 5) that $\left|\sigma_{\mu}^{2}(g)-\sigma_{n}^{2}\right|$ is related to (and in fact somewhat larger than) the quantity $K_{1 / 20}^{n} E_{n}(\theta)=$ $K_{1 / 20}^{n}\left(\prod_{i=1}^{n}\left(1-\theta^{i}\right)\right)^{-1} \theta^{n(n+1) / 2}$, where $\theta \approx 0.860691, K_{1 / 20} \approx 3.631$. We are at liberty to work with any $\kappa \in\left(\theta^{1 / 2}, 1\right)=(0.927734,1)$, and for example with the concrete choice $\kappa=0.95$ we can compute $\sup _{n \in \mathbb{N}} K_{1 / 20}^{n} E_{n}(\theta) / 0.95^{n^{2}} \approx 4.440429 \times 10^{10}$ (the supremum is attained at $n=26$ ), so that $K_{1 / 20}^{n} E_{n}(\theta) \leq C^{\prime} \kappa^{n^{2}}$ for $C^{\prime}=4.5 \times 10^{10}$. It follows, after some additional calculations (along the lines of those detailed in Sect. 8), that the value of $C$ in (3) could be chosen to be of the order of $10^{11}$ when $\kappa=0.95$. 5 This finer analysis consists of using what we call Euler bounds, with the quality of the estimate on $\mid \sigma_{\mu}^{2}(g)-$ $\sigma_{n}^{2} \mid$ closely related to the size of the quantities $K_{t}^{n} E_{n}(\theta)=K_{t}^{n}\left(\prod_{i=1}^{n}\left(1-\theta^{i}\right)\right)^{-1} \theta^{n(n+1) / 2}$ given in Appendix Tables 5 and 6 (for $t=0$ and $t=1 / 20$ respectively), where $\theta=\kappa^{2} \approx 0.860691, K_{0} \approx 3.378, K_{1 / 20} \approx 3.631$. We note that for sufficiently small values of $n$, the quadratic exponential decay of the term $\theta^{n(n+1) / 2}$ is swamped by the exponential increase of the term $K_{t}^{n}$, and the strong increase of $\left(\prod_{i=1}^{n}\left(1-\theta^{i}\right)\right)^{-1}$ (though this latter term is bounded, by $\left.\left(\prod_{i=1}^{\infty}\left(1-\theta^{i}\right)\right)^{-1} \approx 8876.45\right)$. In particular, for $n=20$ both $K_{t}^{n} E_{n}(\theta)$ terms are greater than 1 (hence $n=20$ represents a hopeless case for this naive method), while if $n=25$ then $K_{0}^{n} E_{n}(\theta) \approx 0.000084$ and $K_{1 / 20}^{n} E_{n}(\theta) \approx 0.00051$, which in fact can be used (via arguments similar to those used in the proof of Theorem 3$)$ to justify only a single decimal digit of $\sigma_{\mu}^{2}(g)$. 
small values of $n$ (i.e. for all $1 \leq n \leq P$, where e.g. $P=25$ for the model problem), using exact locations of periodic points (i.e. evaluated to a given precision, typically several hundred decimal places). We next make the observation (see Corollary 1(b)) that $\sigma_{\mu}^{2}(g)$ can be expressed in terms of certain infinite series; it turns out that there are five such series, which for convenience we denote here ${ }^{6}$ as $\sum_{n=1}^{\infty} s_{n}^{(j)}$, for $1 \leq j \leq 5$, where it can be shown that each sequence $\left(s_{n}^{(j)}\right)_{n=1}^{\infty}$ is $O\left(\kappa^{n^{2}}\right)$ as $n \rightarrow \infty$. The error $\left|\sigma_{\mu}^{2}(g)-\sigma_{P}^{2}\right|$ can then be expressed in terms of the tails $\sum_{n=P+1}^{\infty} s_{n}^{(j)}$ of these series, and each of these is $O\left(\kappa^{P^{2}}\right)$ as $P \rightarrow \infty$, a result which incidentally leads to the proof of Theorem 1 . A consequence is that the task of obtaining a concrete bound on $\left|\sigma_{\mu}^{2}(g)-\sigma_{P}^{2}\right|$ reduces to bounding each tail $\sum_{n=P+1}^{\infty} s_{n}^{(j)}$, and here we note that the previously described difficulties in bounding $\left|\sigma_{\mu}^{2}(g)-\sigma_{P}^{2}\right|$ (for e.g. $P=25$ in our model problem) stem from the natural upper bounds on the terms $s_{n}^{(j)}$ being insufficiently sharp for $n \approx P$.

Our resolution of this problem of insufficiently sharp bounds consists of splitting the tails $\sum_{n=P+1}^{\infty} s_{n}^{(j)}$ into two parts, whose estimation can be tackled by distinct methods. Choosing some value ${ }^{7} Q>P$ (e.g. in our model example we take $Q=40$ ) we consider separately the intermediate sum $\sum_{n=P+1}^{Q} s_{n}^{(j)}$ and the deep tail $\sum_{n=Q+1}^{\infty} s_{n}^{(j)}$. The terms in the deep tail can be effectively bounded, essentially by a simple estimate of the form $\left|s_{n}^{(j)}\right| \leq C \kappa^{n^{2}}$, the idea being that $n>Q$ is large enough for the smallness of $\kappa^{n^{2}}$ to dominate the largeness of $C$, to the extent that the whole deep tail is extremely small.

For the purpose of estimating the intermediate (finite) $\operatorname{sum} \sum_{n=P+1}^{Q} s_{n}^{(j)}$ we require some new techniques, whose justification (see Sect. 6) stems from the theory of eigenvalues and approximation numbers applied to a certain auxiliary (transfer) operator; these techniques require a non-trivial amount of computation, though a key point is that the computational effort is relatively light in comparison to that required for locating the $2^{n}$ period- $n$ points for some high value of $n$ (e.g. $n \approx P$ ). The coefficients $s_{n}^{(j)}$ are related to the Taylor series for the determinant of the transfer operator, and can be bounded in terms of the approximation numbers of the operator. These approximation numbers can in turn be bounded by making a judicious choice of basis for an underlying Hilbert space whose inner product is defined by Lebesgue integration, and explicitly computing the norms of the images of (finitely many of) these basis elements under the transfer operator yields a bound on the approximation numbers which implies a bound on the $s_{n}^{(j)}$ for $P+1 \leq n \leq Q$ (see Sect. 7 for further details).

In Sect. 8 we combine all of these various ingredients, in the context of the model problem, to obtain the following rigorous bound on the diffusion coefficient, noting that it represents a significant improvement ${ }^{8}$ on the estimate $0.3458 \leq \sigma_{\mu}^{2}(g) \leq 0.4152$ established in [2] for the same combination of function $g(x)=x^{2}$ and Lanford map $T$.

\footnotetext{
6 In terms of the later notation, these series correspond (see Corollary 1(b)) to $\sum_{n=1}^{\infty} n c_{n}(0), \sum_{n=1}^{\infty} n(n-$ 1) $c_{n}(0), \sum_{n=1}^{\infty} c_{n}^{\prime}(0), \sum_{n=1}^{\infty} n c_{n}^{\prime}(0)$, and $\sum_{n=1}^{\infty} c_{n}^{\prime \prime}(0)$, which themselves correspond to partial derivatives of the determinant of a (transfer) operator.

7 As will become clear, one virtue of this method is that it perfectly feasible, from a computational point of view, to choose $Q$ rather large (e.g. some value well over 100), a choice which may be important for expanding maps $T$ for which the expansion is rather mild, corresponding to significant inertia in the quadratic exponential decay of the terms $s_{n}^{(j)}$, stemming from a value $\kappa \in(0,1)$ being close to 1 .

8 While the rigorous estimate of [2] is less accurate than that of Theorem 2, the general strategy of [2] is based on Ulam's discretization method [16] and can be applied to a wider class of maps $T$ and functions $g$ for which there is no analyticity assumption (see [2] for details and references, and e.g. [10] for a further guide to the literature on numerical computations in the context of piecewise expanding maps).
} 
Theorem 2 For the Lanford map T, with absolutely continuous invariant probability measure $\mu$, if $g(x)=x^{2}$ then the corresponding diffusion coefficient $\sigma_{\mu}^{2}(g)$ satisfies

$$
0.36010948619916067<\sigma_{\mu}^{2}(g)<0.36010948619916067+10^{-17} .
$$

The organisation of this article is as follows. Section 2 consists of preliminary material drawn from the ergodic theory of expanding maps, thermodynamic formalism, and Hilbert spaces of holomorphic functions. Our algorithm is described in Sect. 3, together with various reformulations of the diffusion coefficient. The rapid convergence of the algorithm is illustrated in Sect. 4 for certain cases where $\sigma_{\mu}^{2}(g)$ is known explicitly, and in Sect. 5 for the model problem, where $\sigma_{\mu}^{2}(g)$ does not have a (known) closed form. The key theoretical tools for deriving rigorous error estimates, based on the theory of eigenvalues and approximation numbers, are developed in Sects. 6 and 7. These tools are then applied in detail to the model problem in Sect. 8, proving a result (Theorem 3) that is slightly stronger than Theorem 2, and concluding with a proof of Theorem 1. Some of the numerical data used in the proof of Theorem 3 is collected as an Appendix.

\section{Preliminaries}

\subsection{Ergodic Theory of Expanding Interval Maps}

Suppose the unit interval $X=[0,1]$ is partitioned as $X=X_{1} \cup \cdots \cup X_{l}, l \geq 2$, where $X_{i}=\left[x_{i-1}, x_{i}\right]$, and $0=x_{0}<x_{1}<\cdots<x_{l}=1$. Given $T: X \rightarrow X$, we shall always assume that $\left.T\right|_{X_{i}}$ is real analytic, for each $1 \leq i \leq l$. We say $T$ is piecewise expanding if there exists $\lambda>1$ such that $\left.\left|T^{\prime}\right|\right|_{X_{i}} \geq \lambda$ for all $1 \leq i \leq l$. We say that $T$ is Markov if there exists a $d \times d$ matrix $A$ (the transition matrix for $T$ ) with each entry either 0 or 1 , such that $T\left(X_{i}\right)=\cup_{j: A(i, j)=1} X_{j}$ for each $1 \leq i \leq l$. The collection $\left\{X_{i}\right\}_{i=1}^{l}$ is called the Markov partition for $T . T$ is topologically mixing if some power of the transition matrix $A$ is a strictly positive matrix.

It is well known (see [9]) that any topologically mixing piecewise $C^{\omega}$ expanding Markov map admits a unique ergodic absolutely continuous invariant probability measure, and we shall denote this measure by $\mu$. Our results are valid for all such maps, though to simplify the exposition we shall always assume that $T$ is a full branch expanding map. In other words, each $\left.T\right|_{X_{i}}$ is assumed to be a surjection onto $X$, or, equivalently, every entry of the corresponding transition matrix $A$ is a 1 . For each $1 \leq i \leq l$ we write $\tau_{i}:=\left(\left.T\right|_{X_{i}}\right)^{-1}$, referring to $\left\{\tau_{i}\right\}_{i=1}^{l}$ as the collection of inverse branches of $T$. Since $T$ is expanding, each inverse branch is a contraction mapping on $X$; indeed the real analyticity ${ }^{9}$ of $T$ ensures that the inverse branches have a holomorphic extension to some common complex neighbourhood of $X$ on which they are all contraction mappings.

Notation 1 Let $\mathcal{O}_{n}:=\left\{\underline{x}=\left(x, T(x), \ldots, T^{n-1}(x)\right) \in X^{n}: T^{n}(x)=x\right\}$ denote the collection of periodic orbits of (not necessarily least) period $n$, considered as ordered $n$ tuples. For $\underline{x} \in \mathcal{O}_{n}$ and $g: X \rightarrow \mathbb{R}$, define

$$
g_{\underline{x}}:=\sum_{i=0}^{n-1} g\left(T^{i} x\right), \quad m_{\underline{x}}:=\left(T^{n}\right)^{\prime}(x)=\prod_{i=0}^{n-1} T^{\prime}\left(T^{i} x\right)=\exp \left(\left(\log T^{\prime}\right)_{\underline{x}}\right),
$$

9 As noted previously, by this we mean that $T$ is piecewise real analytic, i.e. each $\left.T\right|_{X_{i}}$ is real analytic, or in other words each $\tau_{i}$ is real analytic. 
and for $n \geq 1, t \in \mathbb{C}$, define

$$
a_{n}(t):=a_{g, n}(t)=\frac{1}{n} \sum_{\underline{x} \in \mathcal{O}_{n}} \frac{\exp \left(t g_{\underline{x}}\right)}{m_{\underline{x}}-1} .
$$

For a continuous function $f: X \rightarrow \mathbb{R}$, its pressure $P(f)=P(f, T)$ is defined (see e.g. [13]) by

$$
P(f)=P(f, T)=\lim _{n \rightarrow \infty} \frac{1}{n} \log \sum_{\underline{x} \in \mathcal{O}_{n}} e^{f_{\underline{x}}} .
$$

\subsection{The Diffusion Coefficient}

Suppose $g: X \rightarrow \mathbb{R}$ is real analytic. Its diffusion coefficient (or variance) $\sigma_{\mu}^{2}(g)$ is defined by

$$
\sigma_{\mu}^{2}(g)=\lim _{n \rightarrow \infty} \frac{1}{n} \int\left(\sum_{i=0}^{n-1} g \circ T^{i}-n \int g d \mu\right)^{2} d \mu .
$$

The diffusion coefficient can be expressed in terms of pressure as follows:

Lemma 1 Let $T: X \rightarrow X$ be a real analytic expanding interval map with absolutely continuous invariant probability measure $\mu$, and suppose $g: X \rightarrow \mathbb{R}$ is real analytic. If $p(t):=P\left(t g-\log \left|T^{\prime}\right|\right)$, then the integral of $g$ with respect to $\mu$ is given by

$$
\mu(g)=\int g d \mu=p^{\prime}(0)
$$

and the diffusion coefficient is given by

$$
\sigma_{\mu}^{2}(g)=p^{\prime \prime}(0)
$$

Proof For (5) see e.g. [11, p. 60], [13, p.133]), and for (6) see e.g. [11, p. 61], [13, p.133]).

\subsection{Holomorphic Extensions}

As noted in Sect. 2.1, the inverse branches of the real analytic expanding map $T$ extend as contraction mappings to some common (simply connected) complex neighbourhood $U$ of $X$. If $g: X \rightarrow \mathbb{R}$ is real analytic then $U$ may be chosen so that $g$ is holomorphic on a neighbourhood of $\bar{U}$. By the Riemann mapping theorem, no generality is lost by assuming that $U$ can be chosen to be a disc $D$, and henceforth we make this assumption: an open disc $D \subset \mathbb{C}$ containing $X$ will be called admissible (for the map $T$ and function $g$ ) if $g$ has a holomorphic extension to a neighbourhood of $\bar{D}$, and each inverse branch $\tau_{i}$ has a holomorphic extension to $D$ such that $\overline{\cup_{i=1}^{l} \tau_{i}(D)} \subset D$. This will allow consideration of transfer operators acting on certain Hilbert spaces of holomorphic functions.

Let $D \subset \mathbb{C}$ be an open disc of radius $r$, centred at $c$. The Hardy space $H^{2}(D)$ consists of those holomorphic functions $\varphi: D \rightarrow \mathbb{C}$ with $\sup _{\varrho<r} \int_{0}^{1}\left|\varphi\left(c+\varrho e^{2 \pi i t}\right)\right|^{2} d t<\infty$. This is a Hilbert space, with inner product given by $(\varphi, \psi)=\int_{0}^{1} \varphi\left(c+r e^{2 \pi i t}\right) \overline{\psi\left(c+r e^{2 \pi i t}\right)} d t$, which is well-defined since members of $H^{2}(D)$ extend as $L^{2}$ functions on the boundary $\partial D$; the norm of $\varphi \in H^{2}(D)$ will be written as $\|\varphi\|=(\varphi, \varphi)^{1 / 2}$. Equivalently, $H^{2}(D)$ is the set of those holomorphic functions $\varphi$ on $D$ such that if $m_{k}(z)=r^{-k}(z-c)^{k}$ for $k \geq 0$, then $\left\{\left(\varphi, m_{k}\right)\right\} \in l^{2}(\mathbb{C})$ (see e.g. [14]), so that $\|\varphi\|=\left(\sum_{k=0}^{\infty}\left|\left(\varphi, m_{k}\right)\right|^{2}\right)^{1 / 2}$. 


\subsection{Transfer Operators and Determinants}

For a real analytic function $g: X \rightarrow \mathbb{R}$, an important ingredient in our method of approximating the diffusion coefficient $\sigma_{\mu}^{2}(g)$ is the function $\Delta_{g}: \mathbb{C}^{2} \rightarrow \mathbb{C}$ defined by

$$
\Delta_{g}(z, t)=\exp \left(-\sum_{n=1}^{\infty} a_{g, n}(t) z^{n}\right)
$$

for sufficiently small values of $z$, and by analytic continuation to the whole of $\mathbb{C}^{2}$. It can be shown that (7) defines an entire function (see Corollary 4), with Taylor series expansion

$$
\Delta_{g}(z, t)=1+\sum_{n=1}^{\infty} c_{g, n}(t) z^{n}=1+\sum_{n=1}^{\infty} c_{n}(t) z^{n}
$$

(where we write $c_{n}(t)$ for $c_{g, n}(t)$ whenever $g$ is understood), from which we deduce the recurrence relation

$$
c_{N}(t)=c_{g, N}(t)=-\frac{1}{N} \sum_{k=0}^{N-1} a_{g, k}(t) \cdot c_{g, N-k}(t) .
$$

For $T$ and $g$ with holomorphic extensions to $D$ as in Sect. 2.3, the corresponding transfer operator $\mathcal{L}_{g, t}: H^{2}(D) \rightarrow H^{2}(D)$ is defined by

$$
\mathcal{L}_{g, t} v(z)=\sum_{T y=z} v(y) e^{f(y)},
$$

for $z \in X$, and by holomorphic continuation for $z \in D$, where $f=\operatorname{tg}-\log \left|T^{\prime}\right|$. The function $\Delta_{g}$ is the determinant $\operatorname{det}\left(I-z \mathcal{L}_{g, t}\right)$ (see [12]), and its zeros are precisely the reciprocals of the eigenvalues of $\mathcal{L}_{g, t}$. The leading (i.e. largest in modulus) eigenvalue of $\mathcal{L}_{g, t}$ is $e^{p(t)}=e^{P\left(t g-\log \left|T^{\prime}\right|\right)}$.

\section{The algorithm}

\subsection{The Diffusion Coefficient in Terms of Derivatives of the Determinant}

The reformulation (6) of the diffusion coefficient $\sigma_{\mu}^{2}(g)$ in terms of pressure, together with the fact that $e^{-p(t)}$ is a zero of $\Delta_{g}(\cdot, t)$, suggests the possibility of representing $\sigma_{\mu}^{2}(g)$ in terms of partial derivatives of $\Delta_{g}$. In order to establish such a representation, as Proposition 1 below, we first adopt the following notational conventions:

Notation 2 We write first partial derivatives as

$$
D_{1} \Delta_{g}(z, t)=\frac{\partial}{\partial z} \Delta_{g}(z, t), \quad D_{2} \Delta_{g}(z, t)=\frac{\partial}{\partial t} \Delta_{g}(z, t),
$$

and second partial derivatives as

$D_{11} \Delta_{g}(z, t)=\frac{\partial^{2}}{\partial z^{2}} \Delta_{g}(z, t), \quad D_{22} \Delta_{g}(z, t)=\frac{\partial^{2}}{\partial t^{2}} \Delta_{g}(z, t), \quad D_{12} \Delta_{g}(z, t)=\frac{\partial^{2}}{\partial z \partial t} \Delta_{g}(z, t)$. 
Proposition 1 If $T: X \rightarrow X$ is a real analytic expanding map with absolutely continuous invariant probability measure $\mu$, the function $g: X \rightarrow \mathbb{R}$ is real analytic, and the determinant $\Delta_{g}$ is defined by (7), then the diffusion coefficient $\sigma_{\mu}^{2}(g)$ can be expressed as

$$
\sigma_{\mu}^{2}(g)=\mu(g)^{2}+\frac{D_{11} \Delta_{g}(1,0) \mu(g)^{2}-2 D_{12} \Delta_{g}(1,0) \mu(g)+D_{22} \Delta_{g}(1,0)}{D_{1} \Delta_{g}(1,0)} .
$$

Proof Let $z(t)=e^{-p(t)}$ where $p(t)=P\left(\operatorname{tg}-\log \left|T^{\prime}\right|\right)$, so that $p(0)=0$ and therefore

$$
z(0)=1 \text {. }
$$

Differentiating gives

$$
z^{\prime}(t)=-p^{\prime}(t) e^{-p(t)}
$$

so Lemma 1 gives

$$
z^{\prime}(0)=-\mu(g)
$$

Differentiating again gives

$$
z^{\prime \prime}(t)=p^{\prime}(t)^{2} e^{-p(t)}-p^{\prime \prime}(t) e^{-p(t)},
$$

so evaluating at $t=0$ and using Lemma 1 gives

$$
z^{\prime \prime}(0)=z^{\prime}(0)^{2}-\sigma_{\mu}^{2}(g),
$$

or in other words

$$
z^{\prime \prime}(0)=\left(\int g d \mu\right)^{2}-\sigma_{\mu}^{2}(g)
$$

The zeros of $\Delta_{g}(\cdot, t)$ are the reciprocals of the eigenvalues of $\mathcal{L}_{g, t}$, and since $e^{p(t)}=z(t)^{-1}$ is the leading eigenvalue of $\mathcal{L}_{g, t}$ then

$$
\Delta_{g}(z(t), t)=0,
$$

so differentiating (15) with respect to $t$ gives

$$
D_{1} \Delta_{g}(z(t), t) z^{\prime}(t)+D_{2} \Delta_{g}(z(t), t)=0,
$$

and therefore

$$
z^{\prime}(0)=-\frac{D_{2} \Delta_{g}(1,0)}{D_{1} \Delta_{g}(1,0)}
$$

Combining (12) and (17) gives

$$
\mu(g)=\frac{D_{2} \Delta_{g}(1,0)}{D_{1} \Delta_{g}(1,0)} .
$$

Differentiating (16) with respect to $t$ gives

$$
D_{1} \Delta_{g}(z(t), t) z^{\prime \prime}(t)+D_{11} \Delta_{g}(z(t), t) z^{\prime}(t)^{2}+2 D_{12} \Delta_{g}(z(t), t) z^{\prime}(t)+D_{22} \Delta_{g}(z(t), t)=0
$$

and evaluating this at $t=0$ then using (11), (12) and (14), gives

$D_{1} \Delta_{g}(1,0)\left(\mu(g)^{2}-\sigma_{\mu}^{2}\right)+D_{11} \Delta_{g}(1,0) \mu(g)^{2}-2 D_{12} \Delta_{g}(1,0) \mu(g)+D_{22} \Delta_{g}(1,0)=0$, 
in other words

$$
\sigma_{\mu}^{2}=\mu(g)^{2}+\frac{D_{11} \Delta_{g}(1,0) \mu(g)^{2}-2 D_{12} \Delta_{g}(1,0) \mu(g)+D_{22} \Delta_{g}(1,0)}{D_{1} \Delta_{g}(1,0)},
$$

which is the required expression (10).

Definition 1 If $g: X \rightarrow \mathbb{R}$ is real analytic, with $\Delta_{g}(z, t)=1+\sum_{n=1}^{\infty} c_{n}(t) z^{n}$, then for $N \geq 1$ define

$$
\begin{aligned}
\sigma_{N}^{2}:= & \left(\frac{\sum_{n=1}^{N} c_{n}^{\prime}(0)}{\sum_{n=1}^{N} n c_{n}(0)}\right)^{2} \\
& +\frac{\sum_{n=1}^{N} n(n-1) c_{n}(0)\left(\frac{\sum_{n=1}^{N} c_{n}^{\prime}(0)}{\sum_{n=1}^{N} n c_{n}(0)}\right)^{2}-2 \sum_{n=1}^{N} n c_{n}^{\prime}(0)\left(\frac{\sum_{n=1}^{N} c_{n}^{\prime}(0)}{\sum_{n=1}^{N} n c_{n}(0)}\right)+\sum_{n=1}^{N} c_{n}^{\prime \prime}(0)}{\sum_{n=1}^{N} n c_{n}(0)} .
\end{aligned}
$$

Corollary 1 Under the same hypotheses as Proposition 1,

(a) The diffusion coefficient $\sigma_{\mu}^{2}(g)$ can be expressed as

$$
\begin{aligned}
\sigma_{\mu}^{2}(g)= & \left(\frac{D_{2} \Delta_{g}(1,0)}{D_{1} \Delta_{g}(1,0)}\right)^{2} \\
& +\frac{D_{11} \Delta_{g}(1,0)\left(\frac{D_{2} \Delta_{g}(1,0)}{D_{1} \Delta_{g}(1,0)}\right)^{2}-2 D_{12} \Delta_{g}(1,0)\left(\frac{D_{2} \Delta_{g}(1,0)}{D_{1} \Delta_{g}(1,0)}\right)+D_{22} \Delta_{g}(1,0)}{D_{1} \Delta_{g}(1,0)} .
\end{aligned}
$$

(b) The diffusion coefficient $\sigma_{\mu}^{2}(g)$ can be expressed as

$$
\begin{aligned}
\sigma_{\mu}^{2}(g)= & \left(\frac{\sum_{n=1}^{\infty} c_{n}^{\prime}(0)}{\sum_{n=1}^{\infty} n c_{n}(0)}\right)^{2} \\
& +\frac{\sum_{n=1}^{\infty} n(n-1) c_{n}(0)\left(\frac{\sum_{n=1}^{\infty} c_{n}^{\prime}(0)}{\sum_{n=1}^{\infty} n c_{n}(0)}\right)^{2}-2 \sum_{n=1}^{\infty} n c_{n}^{\prime}(0)\left(\frac{\sum_{n=1}^{\infty} c_{n}^{\prime}(0)}{\sum_{n=1}^{\infty} n c_{n}(0)}\right)+\sum_{n=1}^{\infty} c_{n}^{\prime \prime}(0)}{\sum_{n=1}^{\infty} n c_{n}(0)} .
\end{aligned}
$$

(c) The sequence of approximations (20) converges, with $\sigma_{N}^{2} \rightarrow \sigma_{\mu}^{2}(g)$ as $N \rightarrow \infty$.

(d) If $g: X \rightarrow \mathbb{R}$ is real analytic such that $\int g d \mu=0$, then $\sigma_{\mu}^{2}(g)$ can be expressed as

$$
\sigma_{\mu}^{2}(g)=\frac{D_{22} \Delta_{g}(1,0)}{D_{1} \Delta_{g}(1,0)}=\frac{\sum_{n=1}^{\infty} c_{n}^{\prime \prime}(0)}{\sum_{n=1}^{\infty} n c_{n}(0)} .
$$

(e) If $g: X \rightarrow \mathbb{R}$ is real analytic such that $\int g d \mu=0$, and $\hat{\sigma}_{N}^{2}$ is defined by

$$
\hat{\sigma}_{N}^{2}:=\frac{\sum_{n=1}^{N} c_{n}^{\prime \prime}(0)}{\sum_{n=1}^{N} n c_{n}(0)},
$$

then $\hat{\sigma}_{N}^{2} \rightarrow \sigma_{\mu}^{2}(g)$ as $N \rightarrow \infty$.

Proof Part (a) follows from Proposition 1, by substituting (18) into (10). Since the Taylor series around 0 for $\Delta_{g}(\cdot, t)$ is written (cf. (8)) as $\Delta_{g}(z, t)=1+\sum_{n=1}^{\infty} c_{n}(t) z^{n}$, termwise differentiation yields (b). Part (d) is a special case of formula (10) in Proposition 1, together with (b), while parts (c) and (e) follow directly from the definitions of $\sigma_{N}^{2}$ and $\hat{\sigma}_{N}^{2}$. 
Remark 1 A consequence of Corollary 1 is that if $g$ is known to have integral zero with respect to the absolutely continuous invariant probability measure $\mu$, then there is a choice of sequence of approximants to the corresponding diffusion coefficient: both the sequence $\sigma_{N}^{2}$ and the sequence $\hat{\sigma}_{N}^{2}$ converge to $\sigma_{\mu}^{2}(g)$.

\subsection{Periodic Orbit Formulae}

The quantities $\sigma_{N}^{2}$ approximating the diffusion coefficient $\sigma_{\mu}^{2}(g)$ are accessible to us in terms of those periodic points of $T$ of period up to $N$. Recall from (4) that

$$
a_{n}(t)=a_{g, n}(t)=\frac{1}{n} \sum_{\underline{x} \in \mathcal{O}_{n}} \frac{\exp \left(\operatorname{tg}_{\underline{x}}\right)}{m_{\underline{x}}-1}
$$

so that the $k$ th order derivative $a_{n}^{(k)}(t)$ is given by

$$
a_{n}^{(k)}(t)=\frac{1}{n} \sum_{\underline{x} \in \mathcal{O}_{n}} \frac{g_{\underline{x}}^{k} \exp \left(t g_{\underline{x}}\right)}{m_{\underline{x}}-1} .
$$

We are interested in derivatives up to order 2 , evaluated at $t=0$, so for $n \geq 1$ define

$$
\alpha_{n}:=a_{n}(0), \quad \beta_{n}:=a_{n}^{\prime}(0), \quad \gamma_{n}:=a_{n}^{\prime \prime}(0),
$$

in other words

$$
\alpha_{n}=\frac{1}{n} \sum_{\underline{x} \in \mathcal{O}_{n}} \frac{1}{m_{\underline{x}}-1}, \quad \beta_{n}=\frac{1}{n} \sum_{\underline{x} \in \mathcal{O}_{n}} \frac{g_{\underline{x}}}{m_{\underline{x}}-1}, \quad \gamma_{n}=\frac{1}{n} \sum_{\underline{x} \in \mathcal{O}_{n}} \frac{g_{\underline{x}}^{2}}{m_{\underline{x}}-1} .
$$

\subsection{Computer Implementation}

Although in certain special cases (e.g. the doubling map of Sect. 4) the periodic points of $T$ are rational and known explicitly, more generally a non-trivial aspect of our algorithm is to locate these periodic points (to within a specified precision). ${ }^{10}$ For this, note that for $1 \leq i \leq l$ the inverse branch $\tau_{i}: X \rightarrow X_{i}$, defined as $\tau_{i}=\left(\left.T\right|_{X_{i}}\right)^{-1}$, is uniformly contracting. For each $\xi \in\{1, \ldots, l\}^{n}$ the composition $\tau:=\tau_{\xi_{1}} \circ \ldots \circ \tau_{\xi_{n}}$ is also uniformly contracting, and the set of period- $n$ points for $T$ is precisely the set of fixed points of such compositions $\tau$. The fixed point for the contraction mapping $\tau$ can be determined using standard techniques (e.g. choose $x_{0} \in X$ and evaluate $x:=\tau^{k}\left(x_{0}\right)$ for suitably large $k$, such that $|\tau(x)-x|<\delta$, where $\delta$ is appropriately small; provided $\tau(x+\varepsilon)-\tau(x)>\eta$ and $\tau(x)-\tau(x-\varepsilon)>\eta$ for $\varepsilon, \eta>0$ satisfying $\eta>\delta+\varepsilon$, an intermediate value argument guarantees that $x$ is within $\varepsilon$ of the true fixed point of $\tau$ ).

Having located the period- $n$ points of $T$, and formed the collection $\mathcal{O}_{n}$, for all $1 \leq n \leq N$, the calculation of orbit sums $a_{n}(0)$ and their derivatives $a_{n}^{\prime}(0), a_{n}^{\prime \prime}(0)$ is then possible (using (4) and (22)) for $n=1, \ldots, N$. Differentiation of the recurrence relation (9) yields recurrence relations for the derivatives $c_{n}^{\prime}(0)$ and $c_{n}^{\prime \prime}(0)$ which can then be computed for $n=1, \ldots, N$, and substitution into (20) gives the approximant $\sigma_{N}^{2}$.

\footnotetext{
10 Specifically, we say that the chosen precision is $10^{-m}$ if any number $\varepsilon$ such that $|\varepsilon|<10^{-m}$ is assumed to be zero; in particular, if we are working with precision $10^{-m}$ then $x$ is declared to be a point of period $n$ for $T$ if $\left|T^{n}(x)-x\right|<10^{-m}$. In our computer programs the various data $\left(T, g\right.$, and the $\left.\tau_{i}\right)$ are approximated with very high precision, of $10^{-999}$, and this precision is maintained during the process of locating periodic points; the points themselves are computed with guaranteed precision of $10^{-250}$.
} 


\section{Test Cases: Approximation of Known Diffusion Coefficients}

For certain combinations of map $T$ and function $g$, the diffusion coefficient is known exactly. While for these cases there is clearly no need for a numerical algorithm to approximate $\sigma_{\mu}^{2}(g)$, it is nonetheless instructive to consider them, by way of a warm-up exercise.

\subsection{Perfect Approximation of the Diffusion Coefficient via Periodic Orbits}

As a simple first example we describe here an expanding map $T$ and function $g$ whose diffusion coefficient $\sigma_{\mu}^{2}(g)$ is exceedingly well approximated by the sequence $\sigma_{n}^{2}$ : in fact it turns out that each $\sigma_{n}^{2}$ is equal to $\sigma_{\mu}^{2}(g)$.

Let $T: X \rightarrow X$ be the doubling map, defined by $T(x)=2 x(\bmod 1)$ on $[0,1)$ and $T(1)=1$, its absolutely continuous invariant probability measure $\mu$ being Lebesgue measure itself. Consider the function $g: X \rightarrow \mathbb{R}$ defined by $g(x)=2 x-1$, which clearly satisfies $\int g d \mu=0$. In fact $g$ is cohomologous to the function $h$ defined by

$$
h(x)= \begin{cases}-1 & \text { if } x \in[0,1 / 2) \\ +1 & \text { if } x \in[1 / 2,1]\end{cases}
$$

and it is easily seen that $\frac{1}{n} \int\left(\sum_{i=0}^{n-1} h \circ T^{i}\right)^{2} d \mu=1$ for all $n \geq 1$, so the corresponding diffusion coefficient is given by the exact formula

$$
\sigma_{\mu}^{2}(g)=\sigma_{\mu}^{2}(h)=\lim _{n \rightarrow \infty} \frac{1}{n} \int\left(\sum_{i=0}^{n-1} h \circ T^{i}\right)^{2} d \mu=1 .
$$

While the existence of an exact formula for $\sigma_{\mu}^{2}(g)$ means there is no need for numerical approximations, this example has the noteworthy feature that our approximations $\sigma_{n}^{2}$ are perfect for each value of $n$ :

Proposition 2 For $T: X \rightarrow X$ the doubling map, and $g(x)=2 x-1$,

$$
c_{g, n}^{\prime \prime}(0)=n c_{g, n}(0) \text { for all } n \geq 1 \text {, }
$$

so in particular

$$
\sigma_{n}^{2}=\frac{\sum_{i=1}^{n} c_{g, i}^{\prime \prime}(0)}{\sum_{i=1}^{n} i c_{g, i}(0)}=1=\sigma_{\mu}^{2}(g) \text { for all } n \geq 1
$$

Proof If $n \geq 1$ and $\underline{x} \in \mathcal{O}_{n}$ then $m_{\underline{x}}=2^{n}$, and $\mathcal{O}_{n}$ has cardinality $2^{n}$, so $\alpha_{n}=\frac{2^{n}}{n\left(2^{n}-1\right)}$. Since $g(1-x)=-g(x)$, and the set $\mathcal{O}_{n}$ is invariant under $x \mapsto 1-x,(23)$ implies that

$$
\beta_{n}=0 \text { for all } n \geq 1,
$$

while

$$
\gamma_{n}=n \alpha_{n}=\frac{2^{n}}{2^{n}-1} \text { for all } n \geq 1 .
$$

Now $\Delta_{g}(z, t)=\exp \left(-\sum_{n=1}^{\infty} a_{g, n}(t) z^{n}\right)$ for $z$ of sufficiently small modulus, therefore

$$
\frac{\partial^{2}}{\partial t^{2}} \Delta_{g}(z, t)=\left(\sum_{n=1}^{\infty} a_{g, n}^{\prime}(t) z^{n}\right)^{2} \Delta_{g}(z, t)-\left(\sum_{n=1}^{\infty} a_{g, n}^{\prime \prime}(t) z^{n}\right) \Delta_{g}(z, t),
$$


and setting $t=0$, so that $a_{g, n}^{\prime}(0)=\beta_{n}=0$ for all $n \geq 1$ by (26), gives

$$
\left.\frac{\partial^{2}}{\partial t^{2}} \Delta_{g}(z, t)\right|_{t=0}=\left(-\sum_{n=1}^{\infty} \gamma_{n} z^{n}\right) \Delta_{g}(z, 0)
$$

for $z$ of sufficiently small modulus.

Now $\frac{\partial}{\partial z} \Delta_{g}(z, t)=\left(-\sum_{n=1}^{\infty} n a_{g, n}(t) z^{n-1}\right) \Delta_{g}(z, t)$, so that by (27),

$$
\left.\frac{\partial}{\partial z} \Delta_{g}(z, t)\right|_{t=0}=\left(-\sum_{n=1}^{\infty} n \alpha_{n} z^{n-1}\right) \Delta_{g}(z, 0)=\left(-\sum_{n=1}^{\infty} \gamma_{n} z^{n-1}\right) \Delta_{g}(z, 0) .
$$

Comparing (28) and (29), which are valid for $z$ of sufficiently small modulus, gives

$$
\left.\frac{\partial^{2}}{\partial t^{2}} \Delta_{g}(z, t)\right|_{t=0}=\left.z \frac{\partial}{\partial z} \Delta_{g}(z, t)\right|_{t=0}
$$

which is in fact valid for all $z \in \mathbb{C}$, by analytic continuation, since both sides of the equation are entire functions of $z$. Writing $\Delta_{g}(z, t)=1+\sum_{n=1}^{\infty} c_{g, n}(t) z^{n}$ we deduce from (30) that

$$
\sum_{n=1}^{\infty} c_{g, n}^{\prime \prime}(0) z^{n}=\sum_{n=1}^{\infty} n c_{g, n}(0) z^{n}
$$

and the required equality (24), and hence (25), follows by comparing coefficients.

Remark 2 The setting of Proposition 2 allows an explicit illustration of the quadratic exponential decay of the coefficients $c_{n}(0)$ (and hence of the $c_{n}^{\prime \prime}(0)=n c_{n}(0)$ ). Writing

$$
\Delta_{g}(z, 0)=\exp \left(-\sum_{n=1}^{\infty} \frac{z^{n}}{n} \frac{2^{n}}{2^{n}-1}\right)=\exp \left(\sum_{j=0}^{\infty} \log \left(1-\frac{z}{2^{j}}\right)\right)=\prod_{j=0}^{\infty}\left(1-\frac{z}{2^{j}}\right),
$$

we see that

$$
c_{n}(0)=(-1)^{n} \frac{2^{n}}{\prod_{i=1}^{n}\left(2^{i}-1\right)},
$$

and therefore ${ }^{11}$

$$
\left|c_{n}(0)\right|=\frac{2^{n}}{\prod_{i=1}^{n}\left(2^{i}-1\right)} \leq \frac{2^{n}}{\prod_{k=1}^{n-1} 2^{k}}=\frac{2^{n}}{2^{n(n-1) / 2}}=\left(\frac{1}{\sqrt{2}}\right)^{n^{2}-3 n},
$$

so in particular

$$
c_{n}(0)=O\left(\kappa^{n^{2}}\right) \text { as } n \rightarrow \infty, \text { for all } \kappa>\frac{1}{\sqrt{2}} .
$$

\subsection{Rapid Approximation}

Suppose, as in Sect. 4.1, that $T: X \rightarrow X$ is the doubling map, and now define $g: X \rightarrow \mathbb{R}$ by $g(x)=x^{2}$. Clearly the integral of $g$ is known explicitly, namely $\int g d \mu=1 / 3$, and if

$\overline{11 \text { In fact a slight sharpening of (31) gives }\left|c_{n}(0)\right| \leq K(1 / \sqrt{2})^{n^{2}-n} \text { for } K}=\prod_{i=1}^{\infty}\left(1-2^{-i}\right)^{-1} \approx 3.462746$. 
$f=g-1 / 3$ then $\int f d \mu=0$, and $\sigma_{\mu}^{2}(g)=\sigma_{\mu}^{2}(f)$, and the equivalent form of the diffusion coefficient $\sigma_{\mu}^{2}(f)=\int f^{2} d \mu+2 \sum_{n=1}^{\infty} \int f \circ T^{n} f d \mu$ (see e.g. [3]) gives

$$
\sigma_{\mu}^{2}(g)=\int f^{2} d \mu+2 \sum_{n=1}^{\infty} \int f \circ T^{n} f d \mu=\frac{1}{45}\left(4+2 \sum_{n=2}^{\infty} \frac{2^{3+n}-2^{n-1}+1}{4^{n}}\right)=\frac{7}{27} .
$$

More generally, for $T$ the doubling map, we note in passing that there are exact formulae for the diffusion coefficient of monomials $x^{k}$ (e.g. for $g(x)=x^{3}$ it can can be shown that $\left.\sigma_{\mu}^{2}(g)=\frac{2783}{11760}\right)$, and indeed for general polynomials, which can be derived from the following result:

Proposition 3 Let $T: X \rightarrow X$ be the doubling map, and $\mu$ Lebesgue measure. If $B_{k}$ denotes the $k$-th Bernoulli polynomial, then its diffusion coefficient is given by

$$
\sigma_{\mu}^{2}\left(B_{k}\right)=\left(\frac{2^{k}+1}{2^{k}-1}\right) \frac{(k !)^{2}}{(2 k) !}\left|\beta_{2 k}\right|
$$

where $\beta_{2 k}=B_{2 k}(0)$ is the $2 k$-th Bernoulli number.

Proof The Bernoulli polynomial $B_{k}$ is an eigenvector of the Perron-Frobenius operator $\mathcal{L}$, with corresponding eigenvalue $2^{-k}$, since it is readily checked that the generating function

$$
G(x, y)=\frac{y e^{x y}}{e^{y}-1}=\sum_{n=0}^{\infty} B_{n}(x) \frac{y^{n}}{n}
$$

satisfies $\mathcal{L} G(x, y)=G(x, y / 2)$ (see [6]). Now $\sigma_{\mu}^{2}\left(B_{k}\right)=\int B_{k}^{2} d \mu+2 \sum_{n=1}^{\infty} \int B_{k} \circ$ $T^{n} B_{k} d \mu$, and

$$
\int B_{k} \circ T^{n} B_{k} d \mu=\int B_{k} \mathcal{L}^{n} B_{k} d \mu=2^{-k n} \int B_{k}^{2} d \mu=2^{-k n} \frac{(k !)^{2}}{(2 k) !}\left|\beta_{2 k}\right|
$$

since $\int B_{k}^{2} d \mu=\frac{(k !)^{2}}{(2 k) !}\left|\beta_{2 k}\right|$ (see e.g. [1]), so the result follows.

For the purpose of observing the speed of approximation of our algorithm, the first six approximations $^{12} \sigma_{n}^{2}$ are $\sigma_{1}^{2}=1 / 4, \sigma_{2}^{2} \approx 0.200617, \sigma_{3}^{2} \approx 0.321554, \sigma_{4}^{2} \approx 0.191905$, $\sigma_{5}^{2} \approx 0.262566, \sigma_{6}^{2} \approx 0.259167$, which after a slow start show signs of approaching $\sigma^{2}=$ $7 / 27=0.259 \dot{2} 5 \dot{9}$. The successive approximants shown in Table 1 illustrate the quadratic exponential convergence, which as in Sect. 4.1 is $O\left(\kappa^{n^{2}}\right)$ as $n \rightarrow \infty$ for any $\kappa>1 / \sqrt{2}$, with the integer parts $l_{n}$ of $\log _{1 / \sqrt{2}}\left|\sigma_{n}^{2}-\sigma_{\mu}^{2}(g)\right|$ also tabulated, and observed to satisfy $n^{2}-11 \leq l_{n} \leq n^{2}-8$ for $7 \leq n \leq 20$.

12 In this example we could equally well exploit the fact that $\int g d \mu=1 / 3$ is known precisely, and use the approximations $\hat{\sigma}_{n}^{2}$ given in Corollary 1(e), for the function $f=g-1 / 3$ (which has zero mean). For example $\hat{\sigma}_{1}^{2} \approx 0.27777, \hat{\sigma}_{2}^{2} \approx 0.43827, \hat{\sigma}_{3}^{2} \approx 0.38515, \hat{\sigma}_{4}^{2}(g)=0.22228, \hat{\sigma}_{5}^{2} \approx 0.26163, \hat{\sigma}_{6}^{2} \approx 0.25918$, $\hat{\sigma}_{7}^{2} \approx 0.259260, \hat{\sigma}_{8}^{2} \approx 0.2592592530, \hat{\sigma}_{9}^{2} \approx 0.259259259277, \hat{\sigma}_{10}^{2} \approx 0.259259259259232$, and more generally the sequences $\sigma_{n}^{2}$ and $\hat{\sigma}_{n}^{2}$ converge to $\sigma^{2}=7 / 27$ at the same rate. 
Table 1 Quadratic exponential convergence of approximations $\sigma_{n}^{2}$ (formed using periodic points of period up to $n$ ) to the diffusion coefficient $\sigma_{\mu}^{2}(g)=7 / 27$ for $T$ the doubling map and $g(x)=x^{2}$. Convergence is $O\left(\kappa^{n^{2}}\right)$ as $n \rightarrow \infty$ for any $\kappa>1 / \sqrt{2}$, with $l_{n}:=\left[\log _{1 / \sqrt{2}}\left|\sigma_{n}^{2}-\sigma_{\mu}^{2}(g)\right|\right]$ tabulated (note that $n^{2}-11 \leq l_{n} \leq n^{2}-8$ for $7 \leq n \leq 20$ )

\begin{tabular}{|c|c|c|}
\hline$n$ & $\sigma_{n}^{2}$ & $l_{n}$ \\
\hline 7 & 0.25926043769067826947393267112262663116939168486481809969132 & 39 \\
8 & 0.25925925204436123648088652153817588245883502977988965009816 & 54 \\
9 & 0.25925925928044946963297173564707938908379066357005434712266 & 70 \\
10 & 0.25925925925922920165588728570045103044970940476420045080830 & 89 \\
11 & 0.25925925925925927995766665750877478547938238966664497426822 & 110 \\
12 & 0.25925925925925925925231122434472300165656411496230961424753 & 133 \\
13 & 0.25925925925925925925926039994958602742793206134750195100492 & 159 \\
14 & 0.25925925925925925925925925916741839472832935929572240739708 & 186 \\
15 & 0.25925925925925925925925925925926289380219067410638708410596 & 215 \\
16 & 0.25925925925925925925925925925925925918842625999409134711672 & 246 \\
17 & 0.25925925925925925925925925925925925925925994017594431303195 & 280 \\
18 & 0.25925925925925925925925925925925925925925925925602612899772 & 315 \\
19 & 0.25925925925925925925925925925925925925925925925925926685093 & 352 \\
20 & 0.25925925925925925925925925925925925925925925925925925925925 & 392 \\
\hline
\end{tabular}

\section{The Lanford Map: Computed Approximations to the Diffusion Coefficient}

Let $T: X \rightarrow X$ be the Lanford map, introduced in [8] and defined by

$$
T(x)=2 x+\frac{1}{2} x(1-x) \quad(\bmod 1) .
$$

As in [2], we shall be interested in approximating the diffusion coefficient $\sigma_{\mu}^{2}(g)$ where the function $g: X \rightarrow \mathbb{R}$ is defined by $g(x)=x^{2}$. Table 2 gives the sequence of approximations $\sigma_{n}^{2}$ to $\sigma_{\mu}^{2}(g)$, using points of period up to $n=25$.

We note that $\left|\sigma_{25}^{2}-\sigma_{24}^{2}\right| \leq 10^{-50}$, strongly suggesting that $\left|\sigma_{25}^{2}-\sigma_{\mu}^{2}(g)\right| \leq 10^{-50}$, though of course this does not constitute a rigorous proof. The remainder of this article is devoted to the development of techniques for rigorously deriving an error bound for approximations of this kind; the approach is valid in the general context of real analytic $T$ and $g$, and in the particular case of our model problem (the Lanford map $T$, and $g(x)=x^{2}$ ), it turns out that we can rigorously prove $\left|\sigma_{25}^{2}-\sigma_{\mu}^{2}(g)\right|<1.48 \times 10^{-18}$ (see Theorem 3 ).

Notation 3 Writing the Lanford map $T$ as

$$
T(x)= \begin{cases}\frac{5 x}{2}-\frac{x^{2}}{2} & \text { for } x \in X_{0}:=\left[0, \frac{5-\sqrt{17}}{2}\right) \\ \frac{5 x}{2}-\frac{x^{2}}{2}-1 & \text { for } x \in X_{1}:=\left[\frac{5-\sqrt{17}}{2}, 1\right],\end{cases}
$$

we see that its real analytic inverse branches $\tau_{i}: X \rightarrow X_{i}$ are given by ${ }^{13}$

$$
\tau_{0}(x)=\frac{1}{2}(5-\sqrt{25-8 x}), \quad \tau_{1}(x)=\frac{1}{2}(5-\sqrt{25-8(x+1)}) .
$$

\footnotetext{
13 Note that when discussing the Lanford map, our indexing of the intervals $X_{i}$ and inverse branches $\tau_{i}$ differs from that used in the rest of the article (where $i=1, \ldots, l$ for some $l \geq 2$ ).
} 
Table 2 Quadratic exponential convergence of approximations $\sigma_{n}^{2}$ (formed using periodic points of period up to $n)$ to the diffusion coefficient $\sigma_{\mu}^{2}(g)$ for the Lanford map $T(x)=2 x+\frac{1}{2} x(1-x)$ ( $\left.\bmod 1\right)$ with absolutely continuous invariant probability measure $\mu$ and function $g(x)=x^{2}$. Convergence is $O\left(\kappa^{n^{2}}\right)$ as $n \rightarrow \infty$ for some $\kappa<1$, and it appears that $\kappa$ may be chosen to be approximately equal to $\sqrt{2 / 3}$; the quantities $l_{n}:=\left[\log _{\sqrt{2 / 3}}\left|\sigma_{n}^{2}-\sigma_{25}^{2}\right|\right]$ are tabulated

\begin{tabular}{|c|c|c|}
\hline$n$ & $\sigma_{n}^{2}$ & $l_{n}$ \\
\hline 5 & 0.758952899740951117289050379204640628558539620626312810772873 & 4 \\
\hline 6 & 0.197308632855131955295248351242175440134141168057749343595655 & 8 \\
\hline 7 & 0.369358110165438698087894292076038462203989053463605038232170 & 23 \\
\hline 8 & 0.359726860572647647166355095586883486801678160823100080553030 & 38 \\
\hline 9 & 0.360119513886829472800814356315023056103035487654360736238916 & 56 \\
\hline 10 & 0.360109316739022418987224588456622015209559108690908744627894 & 76 \\
\hline 11 & 0.360109488057981893593934226218468806113624400705497746276066 & 99 \\
\hline 12 & 0.360109486185859588343561990599828878966607691239152388199536 & 123 \\
\hline & 0.360109486199222993644688357957828705184562158699412145912511 & 149 \\
\hline & 0.360109486199160481163645430040654615882458267775416396263478 & 178 \\
\hline & 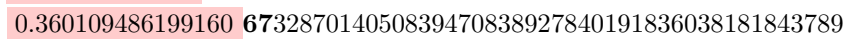 & 209 \\
\hline & $0.36010948619916067 \mathbf{2 8 9 8 3 0 6 0 9 3 6 9 3 5 2 1 7 8 9 6 8 2 0 7 1 8 9 9 1 4 9 1 1 8 3 2 0 8 3 5 6 8 5}$ & 241 \\
\hline & $0.360109486199160672898 \mathbf{8 2 4 6 4 3 2 7 7 2 4 7 0 8 0 5 9 7 4 7 4 5 9 3 2 9 8 6 8 2 5 2 6 0 5 6 6 8 4}$ & 276 \\
\hline & $0.360109486199160672898824 \mathbf{1 8 6 5 6 2 8 2 0 1 3 4 5 5 0 8 8 5 6 2 6 9 3 4 0 5 7 4 6 5 4 5 9 7 2 3}$ & 313 \\
\hline & $0.360109486199160672898824186 \mathbf{8 2 8 6 7 9 0 9 8 9 8 1 5 7 1 3 8 2 2 4 1 7 7 2 6 5 2 0 8 0 3 1 2}$ & 351 \\
\hline & $0.360109486199160672898824186828 \mathbf{5 7 6 7 2 3 1 4 7 9 1 3 7 6 6 7 7 4 7 1 3 7 0 9 9 0 5 5 2 3}$ & 392 \\
\hline & 0.360109486199160672898824186828576749246076750137553904058580 & 435 \\
\hline & $0.36010948619916067289882418682857674924 \mathbf{1 6 6 9 5 0 4 5 3 6 3 1 7 2 5 0 7 4 2 4 9 9}$ & 480 \\
\hline & $0.360109486199160672898824186828576749241669 \mathbf{9 9 7 8 3 3 8 4 0 6 9 0 0 9 0 8 1 8}$ & 527 \\
\hline 24 & $0.360109486199160672898824186828576749241669997 \mathbf{7 9 7 2 2} 7061606200$ & 576 \\
\hline 25 & $0.36010948619916067289882418682857674924166999779722 \mathbf{8 8 6 4 4 1 7 8 8 6}$ & $\infty$ \\
\hline
\end{tabular}

Remark 3 (a) As mentioned in Sect. 3.3, the fact that the $\tau_{i}$ are contractions facilitates the location of the period- $n$ points for $T$, since they are fixed points of suitable compositions $\tau_{\xi_{1}} \circ \cdots \circ \tau_{\xi_{n}}$. The computational procedure for locating the collection of period- $n$ points is very swift for smaller values of $n$; high level software packages such as Mathematica or Matlab may be used for this purpose, though the exponential growth in the number of period- $n$ points makes it advantageous to use an imperative programming language for larger $n$. For this paper the location of periodic points for the Lanford map was carried out on a personal computer with Fortran F07 compiler using MPFUN MPFR packages by D. Bailey (allowing for thread-safe arbitrary precision computations), for all periods $n$ up to $n=25$, following the algorithm as described in Sect. 3 .

(b) It is noteworthy that in all cases studied (those of Sect. 4 as well as this section), the approximations to $\sigma_{\mu}^{2}(g)$ are rather inaccurate (e.g. not correct to 2 decimal places) until points of period at least 5 are incorporated into the approximation.

(c) In $[2, \S 4.6]$, a non-rigorous experiment is performed, which seems to suggest that $\sigma_{\mu}^{2}(g)$ lies in $[0.361,0.363]$. This contrasts with our sequence of approximations in Table 2, and in particular with our best approximation $\sigma_{25}^{2}$. It follows from Theorem 3 that the approximation error of the experiment in [2] is at least $10^{-3}$.

(d) Note that $\min _{x \in X} T^{\prime}(x)=T^{\prime}(1)=3 / 2$, corresponding to the fact that $2 / 3$ is the largest value attained on $X$ by the derivatives of the inverse branches $\tau_{i}$. The value $2 / 3$ appears to be significant concerning the rate at which the approximants $\sigma_{n}^{2}$ approach the diffusion coefficient $\sigma_{\mu}^{2}(g)$. Assuming $\sigma_{25}^{2}$ to be approximately equal to $\sigma_{\mu}^{2}(g)$, so that $\delta_{n}:=$ 
$\left|\sigma_{n}^{2}-\sigma_{25}^{2}\right| \approx\left|\sigma_{n}^{2}-\sigma_{\mu}^{2}(g)\right|$, we note that the values $\varepsilon_{n}:=\exp \left(n^{-2} \log \delta_{n}\right)$ are close to $\sqrt{2 / 3}$ (e.g. $\varepsilon_{22} \approx \sqrt{0.668617}, \varepsilon_{23} \approx \sqrt{0.667478}, \varepsilon_{24} \approx \sqrt{0.666508}$ ), and we therefore tabulate $l_{n}:=\left[\log _{\sqrt{2 / 3}}\left|\sigma_{n}^{2}-\sigma_{25}^{2}\right|\right]$ in Table 2 to illustrate the quadratic exponential convergence. In view of this, it is unsurprising that the value $\sqrt{2 / 3}$ (or some value rather close to it) also appears to dictate the quadratic exponential decay of the coefficients $c_{n}(t)$ of the determinants $\Delta_{g}(z, t)=1+\sum_{n=1}^{\infty} c_{n}(t) z^{n}$ : for example the $c_{n}(0)$ in Appendix Table 7 are such that the terms $\kappa_{n}:=\exp \left(n^{-2} \log \left|c_{n}(0)\right|\right)$ appear to be converging to a value close to, or equal to, $\sqrt{2 / 3}$ (e.g. for $20 \leq n \leq 25$ the $\kappa_{n}$ are approximately $\sqrt{0.674246}$, $\sqrt{0.673346}, \sqrt{0.672570}, \sqrt{0.671899}, \sqrt{0.671313}, \sqrt{0.670801}$ respectively). On the basis of the observed behaviour for the Lanford map, and for the doubling map in Sect. 4, one might speculate that for more general real analytic maps $T: X \rightarrow X$ and functions $g: X \rightarrow \mathbb{R}$, if $\kappa_{T}:=\left(\inf \left\{m_{\underline{x}}^{1 / n}: \underline{x} \in \mathcal{O}_{n}, n \in \mathbb{N}\right\}\right)^{-1 / 2}$ then $\left|\sigma_{n}^{2}-\sigma_{\mu}^{2}(g)\right|=O\left(\kappa^{n^{2}}\right)$ as $n \rightarrow \infty$ for all $\kappa>\kappa_{T}$, and that $\lim _{n \rightarrow \infty} \exp \left(n^{-2} \log \left|c_{g, n}(t)\right|\right)=\kappa_{T}$ for all $t \in \mathbb{C}$. This would constitute a strengthening of our results that if $\theta \in\left(\kappa_{T}^{2}, 1\right)$ is the contraction ratio for an admissible disc, then $\left|\sigma_{n}^{2}-\sigma_{\mu}^{2}(g)\right|=O\left(\kappa^{n^{2}}\right)$ as $n \rightarrow \infty$ for all $\kappa>\theta^{1 / 2}$, and $\lim \sup _{n \rightarrow \infty} \exp \left(n^{-2} \log \left|c_{g, n}(t)\right|\right) \leq \theta^{1 / 2}$ for all $t \in \mathbb{C}$.

\section{Eigenvalues and Approximation Numbers}

In this section we recall the definition of approximation numbers $s_{n}\left(\mathcal{L}_{g, t}\right)$ for the transfer operator $\mathcal{L}_{g, t}$, and introduce a sequence $\alpha_{n}(t)$ of upper bounds for $s_{n}\left(\mathcal{L}_{g, t}\right)$, which we call approximation bounds. By then defining the associated contraction ratio $\theta \in(0,1)$ we are able to establish (see Corollary 2) the exponential bound $s_{n}\left(\mathcal{L}_{g, t}\right) \leq \alpha_{n}(t) \leq K_{t} \theta^{n}$, for a certain explicit constant $K_{t}>0$, which in particular will facilitate (see Corollary 3 in Sect. 7) a proof of the quadratic exponential decay of the Taylor coefficients for the associated determinant.

Let $D \subset \mathbb{C}$ be an open disc of radius $\varrho$ centred at $c$, and let $\left\{\lambda_{n}(t)\right\}_{n=1}^{\infty}$ denote the eigenvalue sequence for the operator $\mathcal{L}_{g, t}: H^{2}(D) \rightarrow H^{2}(D)$, with the convention that eigenvalues are ordered by decreasing modulus and repeated according to their algebraic multiplicities. The Taylor coefficients $c_{n}(t)$ of $\Delta_{g}(\cdot, t)$ then satisfy (see e.g. [15, Lem. 3.3]) the identity

$$
c_{n}(t)=\sum_{i_{1}<\ldots<i_{n}} \prod_{j=1}^{n} \lambda_{i_{j}}(t) .
$$

For $i \geq 1$, the $i$ th approximation number of $\mathcal{L}_{g, t}: H^{2}(D) \rightarrow H^{2}(D)$ is defined to be the value

$$
s_{i}\left(\mathcal{L}_{g, t}\right):=\inf \left\{\left\|\mathcal{L}_{g, t}-M\right\|: \operatorname{rank}(M)<i\right\},
$$

and the well known relation $\left|\sum_{i_{1}<\ldots<i_{n}} \prod_{j=1}^{n} \lambda_{i_{j}}(t)\right| \leq \sum_{i_{1}<\ldots<i_{n}} \prod_{j=1}^{n} s_{i_{j}}\left(\mathcal{L}_{g, t}\right)$ (see e.g. [7, Cor. VI.2.6]) implies that

$$
\left|c_{n}(t)\right| \leq \sum_{i_{1}<\ldots<i_{n}} \prod_{j=1}^{n} s_{i_{j}}\left(\mathcal{L}_{g, t}\right) .
$$

If, for $k \geq 0$, we define $m_{k}: D \rightarrow \mathbb{C}$ by

$$
m_{k}(z)=\varrho^{-k}(z-c)^{k},
$$


then $\left\{m_{k}\right\}_{k=0}^{\infty}$ constitutes an orthonormal basis for $H^{2}(D)$. For $n \geq 1$ we can define the corresponding $n$th approximation bound $\alpha_{n}(t)$ by

$$
\alpha_{n}(t)=\left(\sum_{k=n-1}^{\infty}\left\|\mathcal{L}_{g, t}\left(m_{k}\right)\right\|^{2}\right)^{1 / 2},
$$

and these values yield a simple upper bound on the approximation numbers of the transfer operator:

Lemma 2 For $n \geq 1$, the nth approximation number of $\mathcal{L}_{g, t}: H^{2}(D) \rightarrow H^{2}(D)$ satisfies

$$
s_{n}\left(\mathcal{L}_{g, t}\right) \leq \alpha_{n}(t)
$$

Proof If $f \in H^{2}(D)$ then $\left\{\left(f, m_{k}\right)\right\}_{k=0}^{\infty} \in l^{2}(\mathbb{C})$. Defining $\mathcal{L}_{g, t}^{(n)}:=\mathcal{L}_{g, t} P_{n}$, where $P_{n}$ : $H^{2}(D) \rightarrow H^{2}(D)$ is defined by $P_{n}(f)=\sum_{k=0}^{n-2}\left(f, m_{k}\right) m_{k}$, we obtain the estimate

$$
\left\|\left(\mathcal{L}_{g, t}-\mathcal{L}_{g, t}^{(n)}\right) f\right\|=\left\|\sum_{k=n-1}^{\infty}\left(f, m_{k}\right) \mathcal{L}_{g, t}\left(m_{k}\right)\right\| \leq \sum_{k=n-1}^{\infty}\left|\left(f, m_{k}\right)\right|\left\|\mathcal{L}_{g, t}\left(m_{k}\right)\right\|,
$$

and the Cauchy-Schwarz inequality then implies

$$
\begin{aligned}
\left\|\left(\mathcal{L}_{g, t}-\mathcal{L}_{g, t}^{(n)}\right) f\right\| & \leq\left(\sum_{k=n-1}^{\infty}\left\|\mathcal{L}_{g, t}\left(m_{k}\right)\right\|^{2}\right)^{1 / 2}\left(\sum_{k=n-1}^{\infty}\left|\left(f, m_{k}\right)\right|^{2}\right)^{1 / 2} \\
& \leq\left(\sum_{k=n-1}^{\infty}\left\|\mathcal{L}_{g, t}\left(m_{k}\right)\right\|^{2}\right)^{1 / 2}\|f\|,
\end{aligned}
$$

and hence $\left\|\mathcal{L}_{g, t}-\mathcal{L}_{g, t}^{(n)}\right\| \leq\left(\sum_{k=n-1}^{\infty}\left\|\mathcal{L}_{g, t}\left(m_{k}\right)\right\|^{2}\right)^{1 / 2}=\alpha_{n}(t)$. But $\mathcal{L}_{g, t}^{(n)}$ has rank $n-1$, so the required inequality (39) follows.

Definition 2 Let $D^{\prime}$ be the smallest disc, concentric with $D$, such that $\cup_{i=1}^{l} \tau_{i}(D) \subset D^{\prime}$, and $\varrho, \varrho^{\prime}$ the respective radii of $D, D^{\prime}$. The corresponding contraction ratio $\theta=\theta_{D}$ is defined to be $\theta=\theta_{D}:=\varrho^{\prime} / \varrho$.

Lemma 3 Let $D$ be an admissible disc, with contraction ratio $\theta=\theta_{D}$. If $g:(0,1) \rightarrow \mathbb{R}$ has a holomorphic continuation to a bounded function on $D$, and each $\left|\tau_{i}^{\prime}(\cdot)\right|$ has a holomorphic continuation to a bounded function on $D$, then for all $k \geq 0$,

$$
\left\|\mathcal{L}_{g, t}\left(m_{k}\right)\right\| \leq \theta^{k} \sum_{i=1}^{l}\left\|w_{i, t}\right\|_{\infty}
$$

Proof Defining $w_{i, t}: D \rightarrow \mathbb{C}$ by $w_{i, t}(z)=e^{t g\left(\tau_{i}(z)\right)}\left|\tau_{i}^{\prime}(z)\right|$, we can write $\mathcal{L}_{g, t}=$ $\sum_{i=1}^{l} M_{i, t} C_{i}$, where $C_{i}, M_{i, t}: H^{2}(D) \rightarrow H^{2}(D)$ are given by $C_{i} f:=f \circ \tau_{i}$ and $M_{i, t} f:=w_{i, t} f$. It follows that

$$
\left\|\mathcal{L}_{g, t}\left(m_{k}\right)\right\| \leq \sum_{i=1}^{l}\left\|M_{i, t} C_{i}\left(m_{k}\right)\right\| \leq \sum_{i=1}^{l}\left\|w_{i, t}\right\|_{\infty}\left\|C_{i}\left(m_{k}\right)\right\| .
$$

Now each $\tau_{i}(D)$ is contained in the disc $D^{\prime}$, with the same centre $c$ as $D$, and of radius $\varrho^{\prime}=\theta \varrho$, therefore $\left|C_{i}\left(m_{k}\right)(z)\right|=\varrho^{-k}\left|\tau_{i}(z)-c\right|^{k}<\varrho^{-k}\left(\varrho^{\prime}\right)^{k}=\theta^{k}$ for all $z \in D$. It follows that $\left\|C_{i}\left(m_{k}\right)\right\| \leq \theta^{k}$ and combining this with (41) gives the required inequality (40). 
Corollary 2 Under the hypotheses of Lemma 3, if $K_{t}:=\frac{\sum_{i=1}^{l}\left\|w_{i, t}\right\|_{\infty}}{\theta \sqrt{1-\theta^{2}}}$ then

$$
s_{n}\left(\mathcal{L}_{g, t}\right) \leq \alpha_{n}(t) \leq K_{t} \theta^{n} \text { for all } n \geq 1 .
$$

Proof Combining (38) and Lemma 3 gives

$$
\alpha_{n}(t) \leq\left(\sum_{k=n-1}^{\infty} \theta^{2 k}\right)^{1 / 2} \sum_{i=1}^{l}\left\|w_{i, t}\right\|_{\infty}=\frac{\theta^{n-1}}{\sqrt{1-\theta^{2}}} \sum_{i=1}^{l}\left\|w_{i, t}\right\|_{\infty}=K_{t} \theta^{n},
$$

while the inequality $s_{n}\left(\mathcal{L}_{g, t}\right) \leq \alpha_{n}(t)$ is the content of Lemma 2 .

\section{Euler Bounds and Computed Bounds}

In this section we introduce two different kinds of bound on the Taylor series coefficients of the determinant $\Delta_{g}(\cdot, t)$. The first of these, the Euler bound, has a simple closed form and is readily seen to converge to zero at a quadratic exponential rate. This implies the quadratic exponential decay of the Taylor coefficients (see Corollary 3), and hence that the determinant is an entire function (see Corollary 4); importantly, the inequality proved in Corollary 3 is subsequently used in Sect. 8 to rigorously bound one part of the error term in our diffusion coefficient approximation. The second kind of bound on the Taylor coefficients of the determinant is based on the approximation bounds $\alpha_{n}(t)$ introduced in Sect. 6 , and motivated by the recognition (see the comments in Sect. 1) that despite the quadratic exponential decay of the Euler bounds, for practical purposes they may be insufficiently sharp even for moderately large values of $n$. By first defining an upper computed approximation bound $\alpha_{n, N,+}(t)$ (the large integer $N$ plays the role of a proxy for $\infty$ in the definition (38) of $\alpha_{n}(t)$ ), the inequality (36) then motivates our definition of the upper computed Taylor bound (53), and the resulting Taylor coefficient bound in Proposition 4 provides a key ingredient for the validated approximation of the diffusion coefficient $\sigma_{\mu}^{2}(g)$ described in Sect. 8.

Let us write

$$
E_{n}(r):=\frac{r^{n(n+1) / 2}}{\prod_{i=1}^{n}\left(1-r^{i}\right)}=\sum_{i_{1}<\ldots<i_{n}} r^{i_{1}+\ldots+i_{n}} .
$$

In view of the following bound (44), and the fact that the identity in (43) was first given by Euler (cf. [5, Ch. 16]), we shall refer to the quantity $K_{t}^{n} E_{n}(\theta)$ as the Euler bound on the $n^{\text {th }}$ Taylor coefficient of the determinant $\Delta_{g}(\cdot, t)$.

Corollary 3 Under the hypotheses of Lemma 3, if $\Delta_{g}(z, t)=1+\sum_{n=1}^{\infty} c_{n}(t) z^{n}$ then

$$
\left|c_{n}(t)\right| \leq K_{t}^{n} E_{n}(\theta) \text { for all } n \geq 1 .
$$

Proof From (36) and (42), $\left|c_{n}(t)\right| \leq \sum_{i_{1}<\ldots<i_{n}} \prod_{j=1}^{n} s_{i_{j}}\left(\mathcal{L}_{g, t}\right) \leq K_{t}^{n} \sum_{i_{1}<\ldots<i_{n}} \theta^{i_{1}+\ldots+i_{n}}$.

Corollary 4 Under the hypotheses of Lemma 3, if $\Delta_{g}(z, t)=1+\sum_{n=1}^{\infty} c_{n}(t) z^{n}$, and $\kappa \in$ $\left(\theta^{1 / 2}, 1\right)$, then

$$
c_{n}(t)=O\left(\kappa^{n^{2}}\right) \text { as } n \rightarrow \infty
$$

for all $t \in \mathbb{C}$, and in particular the determinant $\Delta_{g}(\cdot, t)$ is an entire function.

Proof The asymptotic (45) is immediate from (44), and this in particular implies that the Taylor coefficients of $\Delta_{g}(\cdot, t)$ tend to zero faster than any exponential, hence the function is entire. 
In order to exploit Lemma 2, which asserts that

$$
s_{n}\left(\mathcal{L}_{g, t}\right) \leq \alpha_{n}(t)=\left(\sum_{k=n-1}^{\infty}\left\|\mathcal{L}_{g, t}\left(m_{k}\right)\right\|^{2}\right)^{1 / 2},
$$

we require a practical means of computing the approximation bound $\alpha_{n}(t)$. This will consist of bounding $\sum_{k=n-1}^{\infty}\left\|\mathcal{L}_{g, t}\left(m_{k}\right)\right\|^{2}$ by the sum of an exactly computed long finite sum $\sum_{k=n-1}^{N}\left\|\mathcal{L}_{g, t}\left(m_{k}\right)\right\|^{2}$ (the $H^{2}(D)$ norms of the summands can be evaluated using numerical integration, ${ }^{14}$ since each $\mathcal{L}_{g, t} m_{k}$ is known in closed form) and a rigorous upper bound on $\sum_{k=N+1}^{\infty}\left\|\mathcal{L}_{g, t}\left(m_{k}\right)\right\|^{2}$ using (40).

With this in mind, for $n, N \in \mathbb{N}$ with $n \leq N$, we define the lower computed approximation bound

$$
\alpha_{g, n, N,-}(t)=\alpha_{n, N,-}(t):=\left(\sum_{k=n-1}^{N}\left\|\mathcal{L}_{g, t}\left(m_{k}\right)\right\|^{2}\right)^{1 / 2},
$$

and the upper computed approximation bound

$$
\alpha_{g, n, N,+}(t)=\alpha_{n, N,+}(t):=\left(\alpha_{n, N,-}(t)^{2}+\left(\sum_{i=1}^{l}\left\|w_{i, t}\right\|_{\infty}\right)^{2} \frac{\theta^{2(N+1)}}{1-\theta^{2}}\right)^{1 / 2} .
$$

Lemma 4 For $t \in \mathbb{C}$, and $n, N \in \mathbb{N}$ with $n \leq N$,

$$
\alpha_{n, N,-}(t) \leq \alpha_{n}(t) \leq \alpha_{n, N,+}(t) \leq K_{t}\left(1+\theta^{2(N+2-n)}\right)^{1 / 2} \theta^{n} .
$$

Proof The inequality $\alpha_{n, N,-}(t) \leq \alpha_{n}(t)$ is clear. To prove $\alpha_{n}(t) \leq \alpha_{n, N,+}(t)$ we use (40) to give $\alpha_{n}(t)^{2}=\sum_{k=n-1}^{N}\left\|\mathcal{L}_{g, t}\left(m_{k}\right)\right\|^{2}+\sum_{k=N+1}^{\infty}\left\|\mathcal{L}_{g, t}\left(m_{k}\right)\right\|^{2} \leq \sum_{k=n-1}^{N}\left\|\mathcal{L}_{g, t}\left(m_{k}\right)\right\|^{2}+$ $\left(\sum_{i=1}^{l}\left\|w_{i, t}\right\|_{\infty}\right)^{2} \frac{\theta^{2(N+1)}}{1-\theta^{2}}$, and the inequality follows. To prove that $\alpha_{n, N,+}(t) \leq K_{t}(1+$ $\left.\theta^{2(N+2-n)}\right)^{1 / 2} \theta^{n}$, note that combining (42) with $\alpha_{n, N,-}(t) \leq \alpha_{n}(t)$ gives $\alpha_{n, N,-}(t) \leq K_{t} \theta^{n}$, so (47) gives

$\alpha_{n, N,+}(t) \leq\left(\left(K_{t} \theta^{n}\right)^{2}+\left(\sum_{i=1}^{l}\left\|w_{i, t}\right\|_{\infty}\right)^{2} \frac{\theta^{2(N+1)}}{1-\theta^{2}}\right)^{1 / 2}=\left(\left(K_{t} \theta^{n}\right)^{2}+K_{t}^{2} \theta^{2(N+2)}\right)^{1 / 2}$,

and the result follows.

The $\alpha_{n, N,+}(t)$ can now be used to give rigorous upper bounds on the Taylor coefficients of $\Delta_{g}(\cdot, t)$. For $t \in \mathbb{C}$, and $n, M, N \in \mathbb{N}$ with $n \leq M \leq N$, define the Taylor bound $\beta_{n, N,+}^{M}(t)$ by

$$
\beta_{g, n, N,+}^{M}(t)=\beta_{n, N,+}^{M}(t):=\sum_{i_{1}<\ldots<i_{n}} \prod_{j=1}^{n} \alpha_{i_{j}, N,+}^{M}(t),
$$

where the sum is over those $\underline{i}=\left(i_{1}, \ldots, i_{n}\right) \in \mathbb{N}^{n}$ which satisfy $i_{1}<i_{2}<\ldots<i_{n}$, and the sequence $\left(\alpha_{n, N,+}^{M}(t)\right)_{n=1}^{\infty}$ is defined by:

$$
\alpha_{n, N,+}^{M}(t):= \begin{cases}\alpha_{n, N,+}(t) & \text { for } 1 \leq n \leq M \\ K_{t} \theta^{n} & \text { for } n>M\end{cases}
$$

14 e.g. for the computations in Sect. 8 these integrals were computed with 70 digit accuracy using Mathematica. 
Note that from (42), (48) and (50) we have $s_{n}\left(\mathcal{L}_{g, t}\right) \leq \alpha_{n, N,+}^{M}(t)$, which combined with (36) establishes that the Taylor bounds $\beta_{n, N,+}^{M}(t)$ are indeed bounds on the modulus of the $n^{\text {th }}$ Taylor coefficient of $\Delta_{g}(\cdot, t)$ :

$$
\left|c_{n}(t)\right| \leq \beta_{n, N,+}^{M}(t) .
$$

As computable approximations to $\beta_{n, N,+}^{M}(t)$ we then define the lower computed Taylor bound by

$$
\beta_{g, n, N,+}^{M,-}(t)=\beta_{n, N,+}^{M,-}(t):=\sum_{i_{1}<\ldots<i_{n} \leq M} \prod_{j=1}^{n} \alpha_{i_{j}, N,+}(t),
$$

and for $Q \in \mathbb{N}$ with $n \leq Q \leq M \leq N$ we define the upper computed Taylor bound by

$$
\begin{aligned}
\beta_{g, n, N,+}^{M,+}(t)= & \beta_{n, N,+}^{M,+}(t):=\beta_{n, N,+}^{M,-}(t) \\
& +\sum_{l=0}^{n-1} \beta_{l, N,+}^{M,-}(t) \theta^{M(n-l)} E_{n-l}(\theta) K_{t}^{n-l}\left(1+\theta^{2(N+2-Q)}\right)^{(n-l) / 2} .
\end{aligned}
$$

In practice the sum on the righthand side of (53) will be extremely small, though is sufficient for the upper computed Taylor bound to be an upper bound on $\left|c_{n}(t)\right|$ :

Proposition 4 For $t \in \mathbb{C}$, and $Q, M, N \in \mathbb{N}$ with $Q \leq M \leq N$,

$$
\left|c_{n}(t)\right| \leq \beta_{n, N,+}^{M,+}(t) \text { for all } 1 \leq n \leq Q .
$$

Proof If $\mathcal{I}_{n}:=\left\{\underline{i}=\left(i_{1}, \ldots, i_{n}\right) \in \mathbb{N}^{n}: i_{1}<\ldots<i_{n}\right\}$ then $\mathcal{I}_{n}=\bigcup_{l=0}^{n} \mathcal{I}_{n}^{(l)}$ is a disjoint union, where the $\mathcal{I}_{n}^{(l)}$ are defined by $\mathcal{I}_{n}^{(l)}=\left\{\underline{i}=\left(i_{1}, \ldots, i_{n}\right) \in \mathcal{I}_{n}: i_{l} \leq M<i_{l+1}\right\}$ for $1 \leq l \leq n-1$ and $\mathcal{I}_{n}^{(0)}=\left\{\underline{i}=\left(i_{1}, \ldots, i_{n}\right) \in \mathcal{I}_{n}: M<i_{1}\right\}, \mathcal{I}_{n}^{(n)}=\left\{\underline{i}=\left(i_{1}, \ldots, i_{n}\right) \in\right.$ $\left.\mathcal{I}_{n}: i_{n} \leq M\right\}$. If we define $\beta_{n, N,+}^{M,(l)}(s):=\sum_{\underline{i} \in \mathcal{I}_{n}^{(l)}} \prod_{j=1}^{n} \alpha_{i_{j}, N,+}^{M}(s)$ for $0 \leq l \leq n$, so that $\beta_{n, N,+}^{M,(n)}(s)=\beta_{n, N,+}^{M,-}(s)$, we obtain

$$
\beta_{n, N,+}^{M}(s)-\beta_{n, N,+}^{M,-}(s)=\sum_{l=0}^{n-1} \beta_{n, N,+}^{M,(l)}(s) .
$$

Setting $J:=K_{t}\left(1+\theta^{2(N+2-Q)}\right)^{1 / 2}$, Lemma 4 gives $\alpha_{n, N,+}(t) \leq J \theta^{n}$ for all $1 \leq$ $n \leq Q$, and this can be used to bound each $\beta_{n, N,+}^{M,(l)}(s)=\sum_{\underline{i} \in \mathcal{I}_{n}^{(l)}} \prod_{j=1}^{n} \alpha_{i_{j}, N,+}^{M}(s) \leq$ $J^{n-l} \sum_{\underline{i} \in \mathcal{I}_{n}^{(l)}} \theta^{i_{l+1}+\ldots+i_{n}} \prod_{j=1}^{l} \alpha_{i_{j}, N,+}^{M}(s), \quad$ or in other words $\beta_{n, N,+}^{M,(l)}(s) \leq J^{n-l}$ $\left(\sum_{\underline{i} \in \mathcal{I}_{l}^{(l)}} \prod_{j=1}^{l} \alpha_{i_{j}, N,+}^{M}(s)\right)\left(\sum_{\underline{l} \in \mathcal{I}_{n-l}} \theta^{(n-l) M} \theta^{\iota_{1}+\ldots+l_{n-l}}\right)$, and therefore $\beta_{n, N,+}^{M,(l)}(s) \leq J^{n-l}$ $\beta_{l, N,+}^{M,-}(s) \theta^{M(n-l)} E_{n-l}(\theta)$, and substituting these bounds into (55) gives

$$
\beta_{n, N,+}^{M}(t)-\beta_{n, N,+}^{M,-}(t) \leq \sum_{l=0}^{n-1} \beta_{l, N,+}^{M,-}(t) \theta^{M(n-l)} E_{n-l}(\theta) J^{n-l} \quad \text { for all } 1 \leq n \leq Q .
$$

Now (53) and (56) together give $\beta_{n, N,+}^{M}(t) \leq \beta_{n, N,+}^{M,+}(t)$, which combined with (51) gives (54). 


\section{Validated Numerics: The Lanford Map}

With the theory of Sects. 6 and 7 in hand, we are finally in a position to rigorously justify the quality of our computed approximation (see Sect. 5) to the diffusion coefficient $\sigma_{\mu}^{2}(g)$ in the case of our model problem, namely the case of $T$ the Lanford map, $\mu$ its absolutely continuous invariant probability measure, and $g: X \rightarrow \mathbb{R}$ the function $g(x)=x^{2}$. In Sect. 8.1 we choose a suitable disc $D$, compute the associated contraction ratio $\theta$ and constants $K_{t}$, and make choices of the natural numbers $M, N, Q$ which arise in connection with the computed Taylor bounds of Sect. 7. In Sect. 8.2 we establish (see Proposition 5) rigorous bounds on the tails of five series which arise in the formula for $\sigma_{\mu}^{2}(g)$ derived in Corollary 1 ; each series represents a certain derivative of the determinant, and the bounds are established via our Euler bounds and computed Taylor bounds on its Taylor coefficients $c_{n}(t)$. In Sect. 8.3 these tail estimates are combined with the exact evaluations of the corresponding truncated series obtained via periodic point calculations (as described in Sect. 5) to prove a rigorous bound on $\sigma_{\mu}^{2}(g)$ (see Theorem 3). In Sect. 8.4 we prove Theorems 1 and 2, which were stated in Sect. 1; Theorem 2 is seen to be a minor variant of Theorem 3, while the more abstract Theorem 1 is established by combining the techniques used to prove Theorem 3 with the Taylor series asymptotic (45) from Corollary 4.

\subsection{Computed Approximation Bounds and Computed Taylor Bounds}

Choosing ${ }^{15} D$ to be the open disc centred at $c=0.664$, of radius $\varrho=0.87$, we note that both image discs $\tau_{0}(D)$ and $\tau_{1}(D)$ are contained in the disc $D^{\prime}$ centred at $c$, of radius $\varrho^{\prime}=\tau_{1}(c+r)-c$, and the corresponding contraction ratio can be computed as

$$
\begin{aligned}
\theta=\frac{\varrho^{\prime}}{\varrho} & =\frac{918-10 \sqrt{2955}}{435} \\
& =0.860691685064194628752049570062144712194108488685514534 \ldots
\end{aligned}
$$

For $i=0,1$ we have

$$
w_{i, t}(z)=e^{t g\left(\tau_{i}(z)\right)} \tau_{i}^{\prime}(z)=e^{t\left(\tau_{i}(z)\right)^{2}} \tau_{i}^{\prime}(z) .
$$

We shall be particularly interested in the choices $t=0$ and $^{16} t=1 / 20$, and in these cases the supremum norm on $D$ for both functions $w_{0, t}$ and $w_{1, t}$ is attained by evaluating at $z=c+\varrho$,

$$
\begin{aligned}
\left\|w_{0,0}\right\|_{\infty} & =\tau_{0}^{\prime}(c+\varrho)=10 \sqrt{\frac{5}{1591}} \\
& =0.56059589378465950773976123712854581727310648803927 \ldots, \\
\left\|w_{1,0}\right\|_{\infty} & =\tau_{1}^{\prime}(c+\varrho) \\
& =10 \sqrt{\frac{5}{591}}=0.91979546023193796360889752457354830828352420883553 \ldots \\
\left\|w_{0,1 / 20}\right\|_{\infty} & =\exp \left(\frac{1}{20} g\left(\tau_{0}(c+\varrho)\right)\right) \tau_{0}^{\prime}(c+\varrho)
\end{aligned}
$$

15 We make this choice so as to minimise the error estimates arising from the computed Taylor bounds.

16 The choice $t=1 / 20$ is close to optimal for the purpose of estimating $c_{n}^{\prime}(0)$ and $c_{n}^{\prime \prime}(0)$ via Cauchy's integral formula in the proof of Proposition 5. This involves, respectively, the integration of $c_{n}(\zeta) \zeta^{-2}$ and $c_{n}(\zeta) \zeta^{-3}$ over a circular contour centred at 0 , and for both integrands there is a tension between the bound on $\left|c_{n}(\zeta)\right|$,which increases with $|\zeta|$, and the bound on $\left|\zeta^{-k}\right|$ (for $k=2,3$ ), which decreases with $|\zeta|$. 


$$
\begin{aligned}
& =0.575158859780423676330133482123073962520 \ldots, \\
\left\|w_{1,1 / 20}\right\|_{\infty} & =\exp \left(\frac{1}{20} g\left(\tau_{1}(c+\varrho)\right)\right) \tau_{1}^{\prime}(c+\varrho) \\
& =1.016328356027323344809430682923765554385 \ldots
\end{aligned}
$$

We can then compute $K_{0}$ and $K_{1 / 20}$ to be

$$
\begin{aligned}
K_{0} & =\frac{\left\|w_{0,0}\right\|_{\infty}+\left\|w_{1,0}\right\|_{\infty}}{h \sqrt{1-\theta^{2}}}=3.378338827972047629989286401578445782815 \ldots \\
K_{1 / 20} & =\frac{\left\|w_{0,0}\right\|_{\infty}+\left\|w_{1,0}\right\|_{\infty}}{h \sqrt{1-\theta^{2}}}=3.6318660202903086618402475203542507816 \ldots
\end{aligned}
$$

We know that $\left|c_{n}(t)\right| \leq K_{t}^{n} E_{n}(\theta)$ for all $n \geq 1$, and will be interested in those $n$ which are large enough for this bound to be effective, for the cases $t=0$ and $t=1 / 20$. It is easily computed that in both of these cases, the Euler bound $K_{t}^{n} E_{n}(\theta)$ does not even become smaller than 1 until $n>20$, and when $n=26$ (the smallest value of $n$ for which we do not have access to the period- $n$ points) it is of the order of $10^{-5}$ for small $t$, so the Euler bound by itself would only permit a bound on $\sigma_{\mu}^{2}(g)$ which is accurate to around 1 decimal place. It is therefore crucial that we use the computed Taylor bounds in order to yield the high accuracy bound on $\sigma_{\mu}^{2}(g)$ given in Theorem 3, and in the proof of that result we use the Euler bounds only for $n>40$.

Henceforth let $Q=40, M=300, N=400$ (so that in particular $Q \leq M \leq N$, as was assumed throughout Sect. 7), and consider the two cases $t=0$ and $t=1 / 20$.

We first evaluate the $H^{2}(D)$ norms of the monomial images $\mathcal{L}_{g, t}\left(m_{k}\right)$ for $0 \leq k \leq N=$ 400. These norms are decreasing in $k$, and Appendix Table 3 contains the first few evaluations, for $0 \leq k \leq 10$. Using these norms $\left\|\mathcal{L}_{g, t}\left(m_{k}\right)\right\|$ we then evaluate, for $1 \leq n \leq M=300$, the upper computed approximation bounds $\alpha_{n, N,+}(t)=\alpha_{n, 400,+}(t)$ defined (cf. (47)) by ${ }^{17}$

$$
\alpha_{n, N,+}(t)=\left(\sum_{k=n-1}^{N}\left\|\mathcal{L}_{g, t}\left(m_{k}\right)\right\|^{2}+\left(\sum_{i=1}^{2}\left\|w_{i, t}\right\|_{\infty}\right)^{2} \frac{\theta^{2(N+1)}}{1-\theta^{2}}\right)^{1 / 2} .
$$

These bounds are decreasing in $n$; Appendix Table 4 contains the first few evaluations, for $1 \leq n \leq 10$.

The upper computed approximation bounds $\alpha_{n, 400,+}(t)$ are then used to form the upper computed Taylor bounds ${ }^{18} \beta_{n, N,+}^{M,+}(t)=\beta_{n, N,+}^{M,-}(t)+\sum_{l=0}^{n-1} J_{Q, N, t}^{n-l} \beta_{l, N,+}^{M,-}(t) \theta^{M(n-l)} E_{n-l}(\theta)$, where

$$
\beta_{n, N,+}^{M,-}(t)=\beta_{n, 400,+}^{300,-}(t)=\sum_{i_{1}<\ldots<i_{n} \leq 300} \prod_{j=1}^{n} \alpha_{i_{j}, 400,+}(t),
$$

which are listed in Appendix Tables 5 and 6.

17 Note that $h \approx 0.860691$ and $N=400$, so $\frac{\theta^{2(N+1)}}{1-\theta^{2}}<2.2 \times 10^{-52}$. Moreover $\sum_{i=1}^{2}\left\|w_{i, t}\right\|_{\infty}<1.7$ for both $t=0$ and $t=1 / 20$, thus $\left(\sum_{i=1}^{2}\left\|w_{i, t}\right\|_{\infty}\right)^{2} \frac{\theta^{2(N+1)}}{1-\theta^{2}}<6.4 \times 10^{-52}$. Combining these bounds with the values taken by $\alpha_{n, N,+}(t)$, it follows that for $1 \leq n \leq 300$, the approximation bound $\alpha_{n}(t)=$ $\left(\sum_{k=n-1}^{\infty}\left\|\mathcal{L}_{g, t}\left(m_{k}\right)\right\|^{2}\right)^{1 / 2}$ agrees with both computed approximation bounds $\alpha_{n, N,-}(t)$ and $\alpha_{n, N,+}(t)$ to well beyond the desired 70 decimal place precision used in these calculations.

18 The difference $\beta_{n, N,+}^{M,+}(t)-\beta_{n, N,+}^{M,-}(t)=\sum_{l=0}^{n-1} J_{Q, N, t}^{n-l} \beta_{l, N,+}^{M,-}(t) \theta^{M(n-l)} E_{n-l}(\theta)$ is small enough that the upper and lower computed Taylor bounds, and the Taylor bound $\beta_{n, N,+}^{M}(t)$, agree to well beyond the 70 decimal place precision used in these computations. 


\subsection{A Tale of Five Tails: The Ingredients for Validating the Diffusion Coefficient}

The following Proposition 5 gives rigorous bounds on the tails of five series appearing in the formula for $\sigma_{\mu}^{2}(g)$ derived in Corollary 1 .

\section{Proposition 5}

$$
\begin{array}{r}
\left|\sum_{n=26}^{\infty} n c_{n}(0)\right| \leq 6.8 \times 10^{-22}=: \varepsilon_{1}, \\
\left|\sum_{n=26}^{\infty} n(n-1) c_{n}(0)\right| \leq 1.7 \times 10^{-20}=: \varepsilon_{2}, \\
\left|\sum_{n=26}^{\infty} c_{n}^{\prime}(0)\right| \leq 3.4 \times 10^{-21}=: \varepsilon_{3}, \\
\left|\sum_{n=26}^{\infty} n c_{n}^{\prime}(0)\right| \leq 8.7 \times 10^{-20}=: \varepsilon_{4}, \\
\left|\sum_{n=26}^{\infty} c_{n}^{\prime \prime}(0)\right| \leq 6.7 \times 10^{-20}=: \varepsilon_{5} .
\end{array}
$$

Proof Now $\left|c_{n}(0)\right| \leq \beta_{n, N,+}^{M,+}(0)$, and $\left|c_{n}(0)\right| \leq K_{0}^{n} E_{n}(\theta)$, therefore

$$
\left|\sum_{n=26}^{\infty} n c_{n}(0)\right| \leq \sum_{n=26}^{40} n \beta_{n, N,+}^{M,+}(0)+\sum_{n=41}^{\infty} n K_{0}^{n} E_{n}(\theta),
$$

and using the values in Appendix Table 5 we readily compute the finite sum ${ }^{19}$

$$
\sum_{n=26}^{40} n \beta_{n, N,+}^{M,+}(0) \in\left(6.761 \times 10^{-22} \pm 10^{-24}\right),
$$

while the closed form expression for the Euler bound $K_{0}^{n} E_{n}(\theta)$ means we also readily compute that

$$
\sum_{n=41}^{\infty} n K_{0}^{n} E_{n}(\theta) \in\left(1.376 \times 10^{-29} \pm 10^{-31}\right) .
$$

Combining (63)-(65) gives

$$
\left|\sum_{n=26}^{\infty} n c_{n}(0)\right| \leq 6.772 \times 10^{-22}
$$

which is the required (58).

In a similar way, Appendix Table 5 gives the finite sum

$$
\sum_{n=26}^{40} n(n-1) \beta_{n, N,+}^{M,+}(0) \in\left(1.691 \times 10^{-20} \pm 10^{-22}\right)
$$

${ }_{19}$ Note that the $n=26$ term dominates, since $26 \times \beta_{26, N,+}^{M,+}(0) \approx 26 \cdot(2.572 \ldots) \cdot 10^{-23} \approx 6.687 \times 10^{-22}$. 
while the closed form expression for $K_{0}^{n} E_{n}(\theta)$ means we also readily compute that

$$
\sum_{n=41}^{\infty} n(n-1) K_{0}^{n} E_{n}(\theta) \in\left(5.505 \cdot 10^{-28} \pm 10^{-30}\right)
$$

so adding the above two quantities gives

$$
\left|\sum_{n=26}^{\infty} n(n-1) c_{n}(0)\right| \leq 1.7 \times 10^{-20},
$$

which is the required bound (59).

Next we require an estimate on the terms $c_{n}^{\prime}(0)$. From Cauchy's integral formula

$$
c_{n}^{\prime}(0)=\frac{1}{2 \pi i} \int_{\Gamma_{p}} \frac{c_{n}(t)}{t^{2}} d t
$$

where $\Gamma_{p}$ is the positively oriented circle of radius $p$ centred at 0 , we see that $\left|c_{n}^{\prime}(0)\right| \leq$ $\frac{1}{p} \max _{t \in \Gamma_{p}}\left|c_{n}(t)\right|$, and making the choice $p=1 / 20$ gives $\left|c_{n}^{\prime}(0)\right| \leq 20 \max _{t \in \Gamma_{1 / 20}}\left|c_{n}(t)\right|$. Therefore

$$
\left|\sum_{n=26}^{\infty} c_{n}^{\prime}(0)\right| \leq 20\left(\sum_{n=26}^{40} \beta_{n, N,+}^{M,+}(1 / 20)+\sum_{n=41}^{\infty} K_{1 / 20}^{n} E_{n}(\theta)\right),
$$

and using the values in Appendix Table 6 we can evaluate the finite sum

$$
20 \sum_{n=26}^{40} \beta_{n, N,+}^{M,+}(1 / 20) \in\left(3.336 \times 10^{-21} \pm 10^{-23}\right),
$$

while the closed form expression for $K_{1 / 20}^{n} E_{n}(\theta)$ allows the computation

$$
20 \sum_{n=41}^{\infty} K_{1 / 20}^{n} E_{n}(\theta) \in\left(1.304 \times 10^{-28} \pm 10^{-30}\right) .
$$

Combining (68)-(70) gives

$$
\left|\sum_{n=26}^{\infty} c_{n}^{\prime}(0)\right| \leq 3.34 \times 10^{-21},
$$

which is the required bound (60).

Similarly,

$$
\left|\sum_{n=26}^{\infty} n c_{n}^{\prime}(0)\right| \leq 20\left(\sum_{n=26}^{40} n \beta_{n, N,+}^{M,+}(1 / 20)+\sum_{n=41}^{\infty} n K_{1 / 20}^{n} E_{n}(\theta)\right),
$$

and the values in Appendix Table 6 give

$$
\left|20 \sum_{n=26}^{40} \beta_{n, N,+}^{M,+}(1 / 20)\right| \leq 8.679 \times 10^{-20},
$$

while the closed form expression for $K_{1 / 20}^{n} E_{n}(\theta)$ allows the computation

$$
20 \sum_{n=41}^{\infty} n K_{1 / 20}^{n} E_{n}(\theta) \in\left(5.35 \cdot 10^{-27} \pm 10^{-29}\right) .
$$


Combining (71)-(73) gives

$$
\left|\sum_{n=26}^{\infty} n c_{n}^{\prime}(0)\right| \leq 8.68 \times 10^{-20}
$$

which is the bound (61).

To bound $c_{n}^{\prime \prime}(0)$, Cauchy's integral formula gives

$$
c_{n}^{\prime \prime}(0)=\frac{1}{2 \pi i} \int_{\Gamma_{p}} \frac{c_{n}(t)}{t^{3}} d t
$$

so $\left|c_{n}^{\prime \prime}(0)\right| \leq \frac{1}{p^{2}} \max _{t \in \Gamma_{p}}\left|c_{n}(t)\right|$, and again choosing $p=1 / 20$ we have

$$
\left|c_{n}^{\prime \prime}(0)\right| \leq 400 \max _{t \in \Gamma_{1 / 20}}\left|c_{n}(t)\right|,
$$

so that

$$
\left|\sum_{n=26}^{\infty} c_{n}^{\prime \prime}(0)\right| \leq 400\left(\sum_{n=26}^{40} \beta_{n, N,+}^{M,+}(1 / 20)+\sum_{n=41}^{\infty} K_{1 / 20}^{n} E_{n}(\theta)\right) .
$$

The values in Appendix Table 6 then give

$$
400 \sum_{n=26}^{40} \beta_{n, N,+}^{M,+}(1 / 20) \in\left(6.673 \times 10^{-20} \pm 10^{-22}\right)
$$

while (70) implies

$$
400 \sum_{n=41}^{\infty} K_{1 / 20}^{n} E_{n}(\theta) \in\left(2.609 \times 10^{-27} \pm 10^{-29}\right) .
$$

Combining (74)-(76) gives

$$
\left|\sum_{n=26}^{\infty} c_{n}^{\prime \prime}(0)\right| \leq 6.68 \times 10^{-20}
$$

which is the required bound (62).

\subsection{The Rigorous Bound on the Diffusion Coefficient}

In the proof of Theorem 3 we shall make repeated use of the following simple lemma, in settings where $A$ and $B$ are quantities which cannot be computed precisely, but where $a$ and $b$ are computable approximations, and errors $\alpha$ and $\beta$ can be derived.

Lemma 5 If $A, B, a, b \in \mathbb{R}$ and $\alpha, \beta>0$ satisfy $|A-a| \leq \alpha$ and $|B-b| \leq \beta$, then

$$
|A B-a b| \leq(|b|+\beta) \alpha+|a| \beta,
$$

and

$$
\left|\frac{1}{A}-\frac{1}{a}\right| \leq \frac{\alpha}{|a|(|a|-\alpha)} .
$$

We can now justify the quality of our computed approximation to $\sigma_{\mu}^{2}(g)$ as follows: 
Theorem 3 If $T$ is the Lanford map, $\mu$ is its absolutely continuous invariant probability measure, and $g(x)=x^{2}$, then the diffusion coefficient $\sigma_{\mu}^{2}(g)$ can be approximated by $\sigma_{25}^{2}$, which is derived using $T$-periodic points of period up to 25 , so that

$$
\left|\sigma_{\mu}^{2}(g)-\sigma_{25}^{2}\right|<1.48 \times 10^{-18} .
$$

Proof For economy of notation, let us write

$$
\begin{aligned}
R_{N} & =\sum_{n=1}^{N} n c_{n}(0), \quad S_{N}=\sum_{n=1}^{N} n(n-1) c_{n}(0), \\
T_{N} & =\sum_{n=1}^{N} c_{n}^{\prime}(0), \quad U_{N}=\sum_{n=1}^{N} n c_{n}^{\prime}(0), \quad V_{N}=\sum_{n=1}^{N} c_{n}^{\prime \prime}(0),
\end{aligned}
$$

where for our purposes $N$ will equal either 25 or $\infty$, so in particular Corollary 1(b) gives

$$
\sigma_{\mu}^{2}(g)=\left(\frac{T_{\infty}}{R_{\infty}}\right)^{2}+\frac{S_{\infty}\left(\frac{T_{\infty}}{R_{\infty}}\right)^{2}-2 U_{\infty}\left(\frac{T_{\infty}}{R_{\infty}}\right)+V_{\infty}}{R_{\infty}} .
$$

Our periodic orbit calculations (as described in Sect. 5) yield the following:

$$
\begin{aligned}
& R_{25}=\sum_{n=1}^{25} n c_{n}(0) \in\left(-0.121872639684483619872 \pm 10^{-20}\right) \\
& S_{25}=\sum_{n=1}^{25} n(n-1) c_{n}(0) \in\left(0.684579623068217935744 \pm 10^{-20}\right) \\
& T_{25}=\sum_{n=1}^{25} c_{n}^{\prime}(0) \in\left(-0.046788840783927713075 \pm 10^{-20}\right) \\
& U_{25}=\sum_{n=1}^{25} n c_{n}^{\prime}(0) \in\left(0.404063585125598237926 \pm 10^{-20}\right) \\
& V_{25}=\sum_{n=1}^{25} c_{n}^{\prime \prime}(0) \in\left(0.183427185483761853214 \pm 10^{-20}\right)
\end{aligned}
$$

Using (78) with $A=R_{\infty}$ and $a=R_{25}$,

$$
\left|\frac{1}{R_{\infty}}-\frac{1}{R_{25}}\right| \leq \frac{\varepsilon_{1}}{\left|R_{25}\right|\left(\left|R_{25}\right|-\varepsilon_{1}\right)}=: \eta_{1} \in\left(4.578 \times 10^{-20} \pm 10^{-22}\right) .
$$

Combining (88) with (85) and (60), and using (77), we obtain

$$
\left|\frac{T_{\infty}}{R_{\infty}}-\frac{T_{25}}{R_{25}}\right| \leq\left(\left|R_{25}\right|^{-1}+\eta_{1}\right) \varepsilon_{3}+\left|T_{25}\right| \eta_{1}=: \eta_{2} \in\left(3.004 \times 10^{-20} \pm 10^{-22}\right) .
$$

Using (77) again gives

$$
\left|\left(\frac{T_{\infty}}{R_{\infty}}\right)^{2}-\left(\frac{T_{25}}{R_{25}}\right)^{2}\right| \leq\left(2\left|\frac{T_{25}}{R_{25}}\right|+\eta_{2}\right) \eta_{2}=: \eta_{3} \in\left(2.306 \times 10^{-20} \pm 10^{-22}\right) .
$$


Using (77) again we see that

$$
\left|S_{\infty}\left(\frac{T_{\infty}}{R_{\infty}}\right)^{2}-S_{25}\left(\frac{T_{25}}{R_{25}}\right)^{2}\right| \leq\left(\left|S_{25}\right|+\varepsilon_{2}\right) \eta_{3}+\left|\frac{T_{25}}{R_{25}}\right|^{2} \varepsilon_{2}=: \eta_{4} \in\left(1.829 \times 10^{-20} \pm 10^{-22}\right) .
$$

Using (77) again we see that

$$
\left|U_{\infty}\left(\frac{T_{\infty}}{R_{\infty}}\right)-U_{25}\left(\frac{T_{25}}{R_{25}}\right)\right| \leq\left(\left|U_{25}\right|+\varepsilon_{4}\right) \eta_{2}+\left|\frac{T_{25}}{R_{25}}\right| \varepsilon_{4}=: \eta_{5} \in\left(4.553 \times 10^{-20} \pm 10^{-22}\right) .
$$

Writing

$$
W_{N}=S_{N}\left(\frac{T_{N}}{R_{N}}\right)^{2}-2 U_{N}\left(\frac{T_{N}}{R_{N}}\right)+V_{N}
$$

we use (91) and (92) to see that

$$
\left|W_{\infty}-W_{25}\right| \leq \eta_{4}+2 \eta_{5}+\varepsilon_{5}=: \eta_{6} \in\left(1.763 \times 10^{-19} \pm 10^{-21}\right),
$$

and hence

$$
\left|\frac{W_{\infty}}{R_{\infty}}-\frac{W_{25}}{R_{25}}\right| \leq\left(\left|R_{25}\right|^{-1}+\eta_{1}\right) \eta_{6}+\left|W_{25}\right| \eta_{1}=: \eta_{7} \in\left(1.437 \times 10^{-18} \pm 10^{-20}\right) .
$$

Now

$$
\sigma_{\mu}^{2}(g)=\left(\frac{T_{\infty}}{R_{\infty}}\right)^{2}+\frac{W_{\infty}}{R_{\infty}}, \quad \sigma_{25}^{2}=\left(\frac{T_{25}}{R_{25}}\right)^{2}+\frac{W_{25}}{R_{25}},
$$

so from (90) and (94) we deduce

$$
\left|\sigma_{\mu}^{2}(g)-\sigma_{25}^{2}\right| \leq \eta_{3}+\eta_{7} \leq 1.48 \times 10^{-18},
$$

and the desired bound (79) follows.

\subsection{Conclusion}

We conclude by proving the two theorems stated in Sect. 1, beginning with Theorem 2, which follows readily from Theorem 3:

Proof of Theorem 2 Our algorithm (see Table 2) gives

$$
\sigma_{25}^{2} \in\left(0.3601094861991606728988 \pm 10^{-20}\right)
$$

and Theorem 3 gives $\left|\sigma_{\mu}^{2}(g)-\sigma_{25}^{2}\right|<1.48 \times 10^{-18}$, therefore

$$
\sigma_{\mu}^{2}(g) \in(0.36010948619916067143,0.36010948619916067435),
$$

which in particular implies the required result.

Finally, the more abstract Theorem 1 can be proved using ideas similar to those used in the proof of Theorem 3:

Proof of Theorem 1 Writing $\Delta_{g}(z, t)=1+\sum_{n=1}^{\infty} c_{n}(t) z^{n}$, the asymptotic (45) implies that each of $c_{n}(0), c_{n}^{\prime}(0)$ and $c_{n}^{\prime \prime}(0)$ is $O\left(\kappa^{n^{2}}\right)$ as $n \rightarrow \infty$, for some $\kappa \in(0,1)$. For the sums defined in (80), (81), it then follows that each of the five tails $\left|R_{\infty}-R_{n}\right|,\left|S_{\infty}-S_{n}\right|,\left|T_{\infty}-T_{n}\right|$, $\left|U_{\infty}-U_{n}\right|,\left|V_{\infty}-V_{n}\right|$ is $O\left(\kappa^{n^{2}}\right)$ as $n \rightarrow \infty$. Using Lemma 5 we then successively deduce, via arguments analogous to those used in the proof of Theorem 3, that the intermediate 
quantities $\left|1 / R_{\infty}-1 / R_{n}\right|,\left|T_{\infty} / R_{\infty}-T_{n} / R_{n}\right|,\left|\left(T_{\infty} / R_{\infty}\right)^{2}-\left(T_{n} / R_{n}\right)^{2}\right|, \mid S_{\infty}\left(T_{\infty} / R_{\infty}\right)^{2}-$ $S_{n}\left(T_{n} / R_{n}\right)^{2}|,| U_{\infty}\left(T_{\infty} / R_{\infty}\right)-U_{n}\left(T_{n} / R_{n}\right)|,| W_{\infty}-W_{n}|,| W_{\infty} / R_{\infty}-W_{n} / R_{n} \mid$ are also all $O\left(\kappa^{n^{2}}\right)$ as $n \rightarrow \infty$. Since

$$
\sigma_{\mu}^{2}(g)=\left(\frac{T_{\infty}}{R_{\infty}}\right)^{2}+\frac{W_{\infty}}{R_{\infty}} \quad, \quad \sigma_{n}^{2}=\left(\frac{T_{n}}{R_{n}}\right)^{2}+\frac{W_{n}}{R_{n}},
$$

we then deduce that $\left|\sigma_{\mu}^{2}(g)-\sigma_{n}^{2}\right|=O\left(\kappa^{n^{2}}\right)$ as $n \rightarrow \infty$, as required.

Open Access This article is distributed under the terms of the Creative Commons Attribution 4.0 International License (http://creativecommons.org/licenses/by/4.0/), which permits unrestricted use, distribution, and reproduction in any medium, provided you give appropriate credit to the original author(s) and the source, provide a link to the Creative Commons license, and indicate if changes were made.

\section{Appendix: Numerical Data for the Model Problem}

Here we include data (presented in truncated form) for various quantities used in the computation of the diffusion coefficient $\sigma_{\mu}^{2}(g)$ for $T$ the Lanford map and $g(x)=x^{2}$.

Table $3 H^{2}(D)$ norms $\left\|\mathcal{L}_{g, t}\left(m_{k}\right)\right\|$ for Lanford map transfer operator $\mathcal{L}_{g, t}$ for $g(x)=x^{2}$, with $t=0$ and $t=1 / 20$, for disc $D$ centred at $c=0.664$, of radius $\varrho=0.87$

\begin{tabular}{lll}
\hline$k$ & $\left\|\mathcal{L}_{g, 0}\left(m_{k}\right)\right\|$ & $\left\|\mathcal{L}_{g, 1 / 20}\left(m_{k}\right)\right\|$ \\
\hline 0 & 1.072230506759545960034369362057 & 1.104172678680527349319786186152 \\
1 & 0.596049717149185539323469146180 & 0.620723147283434643992316098783 \\
2 & 0.369763875353908620799790724929 & 0.391166259479521916734808474235 \\
3 & 0.276119593229594517922547820447 & 0.294425025977306244191853146578 \\
4 & 0.227958491432695605245256206959 & 0.243204075162724802921362562931 \\
5 & 0.190220640114195164926195507651 & 0.202979268290409813682614341952 \\
6 & 0.157555706161007126216732351954 & 0.168328947218208073434709811106 \\
7 & 0.130346940510248254547131671735 & 0.139478565402897511685346267479 \\
8 & 0.108292842592902764391596660436 & 0.116036335936760027044425349805 \\
9 & 0.090419617507249250203174802637 & 0.0969847954086805719183244969924 \\
10 & 0.075776600475413904133208619148 & 0.081344472381130192060647906273 \\
\hline
\end{tabular}

Table 4 Upper computed approximation bounds $\alpha_{n, N,+}(t)$ for Lanford map transfer operator $\mathcal{L}_{g, t}$ for $g(x)=$ $x^{2}$, with $t=0$ and $t=1 / 20$, for $\operatorname{disc} D$ centred at $c=0.664$, of radius $\varrho=0.87$, with $N=400$

\begin{tabular}{lll}
\hline$n$ & $\alpha_{n, N,+}(0)$ & $\alpha_{n, N,+}(1 / 20)$ \\
\hline 1 & 1.373917067043466452425251967170 & 1.427638696131557424132789994351 \\
2 & 0.859051714093795610515690567921 & 0.904961293287001451810486961337 \\
3 & 0.618623134205199498661411971713 & 0.658526929422198922934937003214 \\
4 & 0.495952879472559770705729997203 & 0.529760959508181474382332373956 \\
5 & 0.411979646210643105413017982909 & 0.440409557454526223835417989155 \\
6 & 0.343164909446721117383095192041 & 0.367168021648856750761912948529 \\
7 & 0.285619087510134990387118189296 & 0.305960410455054090995726782844 \\
8 & 0.238231951270665058019616852773 & 0.255493910483664743663872215393 \\
9 & 0.199409472457670207966369603912 & 0.214062766700294085737845205391 \\
10 & 0.167441924108017577833206337586 & 0.179884509699295419905710072835
\end{tabular}


Table 5 Upper computed Taylor bounds $\beta_{n, N,+}^{M,+}(t)$ for Lanford map transfer operator $\mathcal{L}_{g, t}$ with $t=0$, $g(x)=x^{2}, M=300, N=400$, and disc $D$ centred at $c=0.664$, of radius $\varrho=0.87$

\begin{tabular}{|c|c|}
\hline$n$ & $\beta_{n, N,+}^{M,+}(0)$ \\
\hline 0 & 1.00000000000000000000000000000000000000000000000000000000000000000000 \\
\hline 1 & 5.91638169450327064883976456644941924161928830677363819808060713539812 \\
\hline 2 & 15.5930056102352819935534233140316517118382599986298995283355433265137 \\
\hline 3 & 24.4736698639000709743844193449874942721011409850977481543023124773696 \\
\hline 4 & 25.7754808258198078216455439547621024386170650161794423308493443983097 \\
\hline 5 & 19.4469054872453784049349390698326933609483810493437472532611644864792 \\
\hline 6 & 10.9521106238726346156568796927347289533024574453173311671485824407656 \\
\hline 7 & 4.73540458221471556223636638770483729911053037108254251152025488489004 \\
\hline 8 & 1.60412835316935512560742467899726193208922561294836648618663181927698 \\
\hline 9 & 0.43227019694704976367374985472246979377359534967354408439065009492980 \\
\hline 10 & 0.09375908154725806062303320968739024457872407061926883003524399861986 \\
\hline 11 & 0.01652150342101168996424088686783098220114453369280456250511074495407 \\
\hline 12 & 0.00238293237948001749697274680572517696435951680662097428497778020760 \\
\hline 13 & 0.00028304269463672625512060912147671161988441921765835179658913188285 \\
\hline 14 & 0.00002782677155849364747420872094284640017560258716690718789367793928 \\
\hline 15 & $2.27392403136506520702724564410473340446650639579722032205330246 \times 10^{-6}$ \\
\hline 16 & $1.55001252830385066339340115927214700511589047296031298506903537 \times 10^{-7}$ \\
\hline 17 & $8.83999766101668028957629529487694836331978784991495390797963332 \times 10^{-9}$ \\
\hline 18 & $4.22910735620174411941773831057180480980291549806736590593484206 \times 10^{-10}$ \\
\hline 19 & $1.70094619827541573471532294812274420039877275633876944149746895 \times 10^{-11}$ \\
\hline 20 & $5.76253574844793924863326504428979970720822216845679266337327572 \times 10^{-13}$ \\
\hline 21 & $1.64719543361358736487381155317306660980894721531616471482462791 \times 10^{-14}$ \\
\hline 22 & $3.97851343969582857919989434542234955671337278329883396694974188 \times 10^{-16}$ \\
\hline 23 & $8.13015813907035571457013570353731056069789743557218471587901060 \times 10^{-18}$ \\
\hline 24 & $1.40725206079897102652397486145471051771037644337044022147968721 \times 10^{-19}$ \\
\hline 25 & $2.06526294831298446834304647553465436309716315513236215167585825 \times 10^{-21}$ \\
\hline 26 & $2.57215243104667105215824167841697542562499595143189055321291537 \times 10^{-23}$ \\
\hline 27 & $2.72070490994043636692628731754208142998380316685815821651703231 \times 10^{-25}$ \\
\hline 28 & $2.44590093688207448627993621838824589670936482665024996736095949 \times 10^{-27}$ \\
\hline 29 & $1.87002244040068058743646064182298912700729836481886839796879296 \times 10^{-29}$ \\
\hline 30 & $1.21662223061110407539286299080285544487051750134294707706848536 \times 10^{-31}$ \\
\hline 31 & $6.73894859299114408105902864970222134509197580734796373553489611 \times 10^{-34}$ \\
\hline 32 & $3.17951954702135581037427993130825377583766175736565112414287832 \times 10^{-36}$ \\
\hline 33 & $1.27835316671718885749177319622144884560379835925694002589279209 \times 10^{-38}$ \\
\hline 34 & $4.38158222578128011907566552141075137983249232012587406086472299 \times 10^{-41}$ \\
\hline 35 & $1.28073190382903792751606572170641919527714030160004122585114832 \times 10^{-43}$ \\
\hline 36 & $3.19356882118247708913130052922372437303467184375302002671812072 \times 10^{-46}$ \\
\hline
\end{tabular}


Table 5 continued

\begin{tabular}{ll}
\hline$n$ & $\beta_{n, N,+}^{M,+}(0)$ \\
\hline 37 & $6.79543778777729442805037570050636716580086586696826218847684626 \times 10^{-49}$ \\
38 & $1.23425230994365846085219734895268399165400230577981026871766096 \times 10^{-51}$ \\
39 & $1.91403130218947580428297036198227018700547685312967193646613596 \times 10^{-54}$ \\
40 & $2.53488275012643454408402888067456803151862774782787701968600395 \times 10^{-57}$ \\
\hline
\end{tabular}

Table 6 Upper computed Taylor bounds $\beta_{n, N,+}^{M,+}(t)$ for Lanford map transfer operator $\mathcal{L}_{g, t}$ with $t=1 / 20$, $g(x)=x^{2}, M=300, N=400$, and disc $D$ centred at $c=0.664$, of radius $\varrho=0.87$

\begin{tabular}{|c|c|}
\hline$n$ & $\beta_{n, N,+}^{M,+}(1 / 20)$ \\
\hline 0 & 1.00000000000000000000000000000000000000000000000000000000000000000000 \\
\hline 1 & 6.27842519703334406022340033748927178829675608493814980507714608797459 \\
\hline 2 & 17.6003362119988183866754213426824373437028159179692389550954549117423 \\
\hline 3 & 29.4366898216927944152313565661448722215928824631400913757149291269852 \\
\hline 4 & 33.0867611911634920240882205854397496498017624116086418773855567059626 \\
\hline 5 & 26.67533564351103485555307719236605270980278686636067429378252055347670 \\
\hline 6 & 16.0710965899538078436477208145810313529265723398946425024786919332663 \\
\hline 7 & 7.44063666302454054383943558694860088009355060221952940348668203608729 \\
\hline 8 & 2.70127094064217596236351990259358495525841780837765629213799111450263 \\
\hline 9 & 0.78071782310801484862526127910162690666654602643063005600755159227045 \\
\hline 10 & 0.18174627347531441236057904915305258962787959831002794386840974964700 \\
\hline 11 & 0.03439486702274223053770708403705609182708982722820690472000498696375 \\
\hline 12 & 0.00533095477056366674311114131747398500384802945013153524121964534649 \\
\hline 13 & 0.00068082190626498655702769374902264509667584958345813328144351285726 \\
\hline 14 & 0.00007200388275841965360071435691243740847574318817522346898702039135 \\
\hline 15 & $6.33271392110873459655379479015310649620066560990384843859282266 \times 10^{-6}$ \\
\hline 16 & $4.64800848470789807590971030856240671418742805942662945873297418 \times 10^{-7}$ \\
\hline 17 & $2.85553568027041612564729759621742273463462761215038353862022367 \times 10^{-8}$ \\
\hline 18 & $1.47219033472979293213438915492415236429139393490859859980761806 \times 10^{-9}$ \\
\hline 19 & $6.38341105718717757915909774574402061895030645318360898190706892 \times 10^{-11}$ \\
\hline 20 & $2.33228685756150438219265787953400833864987726565924390772235464 \times 10^{-12}$ \\
\hline 21 & $7.19235908955625772063753408644833359500811213574185949878327842 \times 10^{-14}$ \\
\hline 22 & $1.87477911111247179179899643900730715712136223446850276009517444 \times 10^{-15}$ \\
\hline 23 & $4.13590334226334088615441884060439848738610606398417273687178495 \times 10^{-17}$ \\
\hline 24 & $7.73070140894702140522209504941473132524640938008515421479045415 \times 10^{-19}$ \\
\hline 25 & $1.22553689288336734377055577029417960323883309163815462793346801 \times 10^{-20}$ \\
\hline 26 & $1.64920824712133774857887341667964355887802022954017778167641175 \times 10^{-22}$ \\
\hline 27 & $1.88541699764734059705504785147513397623798878093845816667050018 \times 10^{-24}$ \\
\hline 28 & $1.83243033954492300016575720796250290917142059356438926786130103 \times 10^{-26}$ \\
\hline
\end{tabular}


Table 6 continued

\begin{tabular}{ll}
\hline$n$ & $\beta_{n, N,+}^{M,+}(1 / 20)$ \\
\hline 29 & $1.51499027117462581296207671180385128334726539966767247928642535 \times 10^{-28}$ \\
30 & $1.06610892290501996421078468465905043866340941985470001595680173 \times 10^{-30}$ \\
31 & $6.38889899955948918993224875553710714558745184415119687576651408 \times 10^{-33}$ \\
32 & $3.26201347689449394139927031534076848195196188267851020944823308 \times 10^{-35}$ \\
33 & $1.41959703603586750328152045570639202099993714044440242822016384 \times 10^{-37}$ \\
34 & $5.26783612982327434661909624961672214739725086799900939911078203 \times 10^{-40}$ \\
35 & $1.66740574038123727102261424406832509052875443488407531926208542 \times 10^{-42}$ \\
36 & $4.50332846038763649055145870908682675097043038881690772288865126 \times 10^{-45}$ \\
37 & $1.03810074880453070195009955535972632680521900405212333475588763 \times 10^{-47}$ \\
38 & $2.04305109106193115584629645721971197363097686254964237369887319 \times 10^{-50}$ \\
39 & $3.43371464442528749462619173440602768944065612071967642619009054 \times 10^{-53}$ \\
40 & $4.92944333332153669605064037563063626241759423036425615345390256 \times 10^{-56}$ \\
\hline
\end{tabular}

Table 7 Lanford map: quantities $E_{n}(\theta)$, Euler bounds $K_{0}^{n} E_{n}(\theta) \geq\left|c_{n}(0)\right|$ and $K_{1 / 20}^{n} E_{n}(\theta) \geq\left|c_{n}(1 / 20)\right|$, for disc $D$ centred at $c=0.664$ of radius $\varrho=0.87$ (so $\theta \approx 0.860691$ ), and coefficients $c_{n}(0)$ of determinant $\Delta_{g}(z, 0)=1+\sum_{n=1}^{\infty} c_{n}(0) z^{n}$ for $1 \leq n \leq 25$

\begin{tabular}{|c|c|c|c|c|}
\hline$n$ & $E_{n}(\theta)$ & $K_{0}^{n} E_{n}(\theta)$ & $K_{1 / 20}^{n} E_{n}(\theta)$ & $c_{n}(0)$ \\
\hline 1 & 6.178322417156 & 20.872466513610 & 22.438839249268 & -2.6666666666666 \\
\hline 2 & 17.65689470317 & 201.52119806742 & 232.90240067474 & 2.7671447514335 \\
\hline 3 & 31.06413807670 & 1197.7575633058 & 1488.1570459342 & -1.4795873463144 \\
\hline 4 & 37.77912144494 & 4921.1281303006 & 6573.1134807116 & 0.4548595107266 \\
\hline 5 & 33.81588669461 & 14881.160574758 & 21368.295194231 & -0.0850094435255 \\
\hline 6 & 23.16343104695 & 34436.746764748 & 53159.612067142 & 0.0099728366537 \\
\hline 7 & 12.46666302384 & 62614.173627336 & 103910.38491503 & -0.0007489690661 \\
\hline 8 & 5.372126671547 & 91153.192091602 & 162624.05897399 & 0.0000364631148 \\
\hline 9 & 1.879626036050 & 107745.78610040 & 206652.10008498 & $-1.160290 \times 10^{-6}$ \\
\hline 10 & 0.539728880137 & 104521.99820457 & 215513.18595829 & $2.426597 \times 10^{-8}$ \\
\hline 11 & 0.128260613487 & 83912.867873119 & 186003.58802690 & $-3.347923 \times 10^{-10}$ \\
\hline 12 & 0.025393003771 & 56124.506215835 & 133743.26007194 & $3.055073 \times 10^{-12}$ \\
\hline 13 & 0.004210820328 & 31441.870270393 & 80547.925161555 & $-1.847222 \times 10^{-14}$ \\
\hline 14 & 0.000587419646 & 14818.127696936 & 40809.937890039 & $7.410060 \times 10^{-17}$ \\
\hline 15 & 0.000069186089 & 5896.1273174416 & 17456.857546576 & $-1.973903 \times 10^{-19}$ \\
\hline 16 & $6.900312 \times 10^{-6}$ & 1986.6439509659 & 6323.3303122469 & $3.493983 \times 10^{-22}$ \\
\hline 17 & $5.842164 \times 10^{-7}$ & 568.23539709633 & 1944.3781298067 & $-4.111689 \times 10^{-25}$ \\
\hline 18 & $4.207596 \times 10^{-8}$ & 138.25848435416 & 508.59355192450 & $3.217996 \times 10^{-28}$ \\
\hline 19 & $2.582298 \times 10^{-9}$ & 28.666012024725 & 113.36342306436 & $-1.675484 \times 10^{-31}$ \\
\hline
\end{tabular}


Table 7 continued

\begin{tabular}{|c|c|c|c|c|}
\hline$n$ & $E_{n}(\theta)$ & $K_{0}^{n} E_{n}(\theta)$ & $K_{1 / 20}^{n} E_{n}(\theta)$ & $c_{n}(0)$ \\
\hline 20 & $1.352474 \times 10^{-10}$ & 5.0721615997794 & 21.563803644473 & $5.804730 \times 10^{-35}$ \\
\hline 21 & $6.052600 \times 10^{-12}$ & 0.7668479758598 & 3.5048398385881 & $-1.338407 \times 10^{-38}$ \\
\hline 22 & $2.316879 \times 10^{-13}$ & 0.0991685458492 & 0.4872585410565 & $2.054103 \times 10^{-42}$ \\
\hline 23 & $7.592813 \times 10^{-15}$ & 0.0109793456797 & 0.0579947377200 & $-2.098636 \times 10^{-46}$ \\
\hline 24 & $2.131922 \times 10^{-16}$ & 0.0010414738636 & 0.0059140789758 & $1.427503 \times 10^{-50}$ \\
\hline 25 & $5.132067 \times 10^{-18}$ & 0.0000846978646 & 0.0005170562827 & $-6.465176 \times 10^{-55}$ \\
\hline 26 & $1.059758 \times 10^{-19}$ & $5.908676 \times 10^{-6}$ & 0.0000387777099 & \\
\hline 27 & $1.878111 \times 10^{-21}$ & $3.537593 \times 10^{-7}$ & $2.495896 \times 10^{-6}$ & \\
\hline 28 & $2.857674 \times 10^{-23}$ & $1.818454 \times 10^{-8}$ & $1.379264 \times 10^{-7}$ & \\
\hline 29 & $3.734497 \times 10^{-25}$ & $8.028327 \times 10^{-10}$ & $6.546314 \times 10^{-9}$ & \\
\hline 30 & $4.192848 \times 10^{-27}$ & $3.045126 \times 10^{-11}$ & $2.669338 \times 10^{-10}$ & \\
\hline 31 & $4.045340 \times 10^{-29}$ & $9.925545 \times 10^{-13}$ & $9.353613 \times 10^{-12}$ & \\
\hline 32 & $3.354789 \times 10^{-31}$ & $2.780787 \times 10^{-14}$ & $2.817211 \times 10^{-13}$ & \\
\hline 33 & $2.391781 \times 10^{-33}$ & $6.697725 \times 10^{-16}$ & $7.294669 \times 10^{-15}$ & \\
\hline 34 & $1.466203 \times 10^{-35}$ & $1.387085 \times 10^{-17}$ & $1.624083 \times 10^{-16}$ & \\
\hline 35 & $7.729370 \times 10^{-38}$ & $2.470337 \times 10^{-19}$ & $3.109479 \times 10^{-18}$ & \\
\hline 36 & $3.504471 \times 10^{-40}$ & $3.783885 \times 10^{-21}$ & $5.120307 \times 10^{-20}$ & \\
\hline 37 & $1.366704 \times 10^{-42}$ & $4.985320 \times 10^{-23}$ & $7.252333 \times 10^{-22}$ & \\
\hline 38 & $4.584986 \times 10^{-45}$ & $5.650148 \times 10^{-25}$ & $8.836315 \times 10^{-24}$ & \\
\hline 39 & $1.323263 \times 10^{-47}$ & $5.508981 \times 10^{-27}$ & $9.262096 \times 10^{-26}$ & \\
\hline 40 & $3.285698 \times 10^{-50}$ & $4.621211 \times 10^{-29}$ & $8.352576 \times 10^{-28}$ & \\
\hline 41 & $7.019503 \times 10^{-53}$ & $3.335322 \times 10^{-31}$ & $6.480805 \times 10^{-30}$ & \\
\hline 42 & $1.290338 \times 10^{-55}$ & $2.071276 \times 10^{-33}$ & $4.326691 \times 10^{-32}$ & \\
\hline 43 & $2.040971 \times 10^{-58}$ & $1.106814 \times 10^{-35}$ & $2.485531 \times 10^{-34}$ & \\
\hline 44 & $2.777937 \times 10^{-61}$ & $5.089363 \times 10^{-38}$ & $1.228667 \times 10^{-36}$ & \\
\hline 45 & $3.253667 \times 10^{-64}$ & $2.013804 \times 10^{-40}$ & $5.226549 \times 10^{-39}$ & \\
\hline
\end{tabular}

\section{References}

1. Apostol, T.: Introduction to analytic number theory. Springer, New York (1976)

2. Bahsoun, W., Galatolo, S., Nisoli, I., Niu, X.: Rigorous approximation of diffusion coefficients for expanding maps. J. Stat. Phys. 163, 1486-1503 (2016)

3. Collet, P.: Some ergodic properties of maps of the interval. In: Dynamical Systems (Temuco, 1991/1992), 55-91, Travaux en Cours, 52, Hermann, Paris (1996)

4. Collet, P., Eckmann, J.-P.: Iterated Maps on the Interval as Dynamical Systems. Birkhauser, Boston (1980)

5. Euler, L.: Introductio in Analysin Infinitorum. Marcum-Michaelem Bousquet, Lausannae (1748)

6. Gaspard, P.: $r$-adic one-dimensional maps and the Euler summation formula. J. Phys. A 25, L483-L485 (1992)

7. Gohberg, I., Goldberg, S., Kaashoek, M.A.: Classes of Linear Operators, vol. 1. Birkhäuser, Berlin (1990)

8. Lanford III, O.E.: Informal remarks on the orbit structure of discrete approximations to chaotic maps. Exp. Math. 7, 317-324 (1998)

9. Lasota, A., Yorke, J.A.: On the existence of invariant measures for piecewise monotonic transformations. Trans. Am. Math. Soc. 186, 481-488 (1973) 
10. Liverani, C.: Rigorous numerical investigation of the statistical properties of piecewise expanding maps. A feasibility study. Nonlinearity 14, 463-490 (2001)

11. Parry, W., Pollicott, M.: Zeta functions and the periodic orbit structure of hyperbolic dynamics. Astérisque 187-188 (1990)

12. Ruelle, D.: Zeta-functions for expanding maps and Anosov flows. Invent. Math. 34, 231-242 (1976)

13. Ruelle, D.: Thermodynamic Formalism. Addison-Wesley, Reading, MA (1978)

14. Shapiro, J.H.: Composition Operators and Classical Function Theory. Springer, New York (1993)

15. Simon, B.: Trace Ideals and Their Applications. LMS Lecture Note Series, vol. 35. Cambridge University Press, Cambridge (1979)

16. Ulam, S.: Problems in Modern Mathematics. Interscience, New York (1960) 